\title{
Spatial Arrangement Drastically Changes the Neural Representation of Multiple Visual Stimuli That Compete in More Than One Feature Domain
}

\author{
Steven Wiesner, ${ }^{\star}$ Ian W. Baumgart, ${ }^{\star}$ and $\odot$ Xin Huang \\ Department of Neuroscience, School of Medicine and Public Health, Physiology Graduate Training Program, McPherson Eye Research Institute, University \\ of Wisconsin-Madison, Madison, Wisconsin 53705
}

\begin{abstract}
Natural scenes often contain multiple objects and surfaces. However, how neurons in the visual cortex represent multiple visual stimuli is not well understood. Previous studies have shown that, when multiple stimuli compete in one feature domain, the evoked neuronal response is biased toward the stimulus that has a stronger signal strength. We recorded from two male macaques to investigate how neurons in the middle temporal cortex (MT) represent multiple stimuli that compete in more than one feature domain. Visual stimuli were two random-dot patches moving in different directions. One stimulus had low luminance contrast and moved with high coherence, whereas the other had high contrast and moved with low coherence. We found that how MT neurons represent multiple stimuli depended on the spatial arrangement. When two stimuli were overlapping, MT responses were dominated by the stimulus component that had high contrast. When two stimuli were spatially separated within the receptive fields, the contrast dominance was abolished. We found the same results when using contrast to compete with motion speed. Our neural data and computer simulations using a V1-MT model suggest that the contrast dominance found with overlapping stimuli is due to normalization occurring at an input stage fed to MT, and MT neurons cannot overturn this bias based on their own feature selectivity. The interaction between spatially separated stimuli can largely be explained by normalization within MT. Our results revealed new rules on stimulus competition and highlighted the impact of hierarchical processing on representing multiple stimuli in the visual cortex.
\end{abstract}

Key words: neural encoding; receptive field; normalization; visual cortex; segmentation; stimulus competition

Significance Statement

Previous studies have shown that the neural representation of multiple visual stimuli can be accounted for by a divisive normalization model. By using multiple stimuli that compete in more than one feature domain, we found that luminance contrast has a dominant effect in determining competition between multiple stimuli when they are overlapping but not spatially separated. Our results revealed that neuronal responses to multiple stimuli in a given cortical area cannot be simply predicted by the population neural responses elicited in that area by the individual stimulus components. To understand the neural representation of multiple stimuli, rather than considering response normalization only within the area of interest, one must consider the computations including normalization occurring along the hierarchical visual pathway.

\section{Introduction}

In natural scenes, multiple visual stimuli are often present in a local spatial region. Although it is generally well understood how

Received Aug. 11, 2019; revised Dec. 15, 2019; accepted Jan. 7, 2020.

Author contributions: S.W., I.W.B., and X.H. designed research; S.W. and I.W.B. performed research; S.W., I.W.B., and X.H. analyzed data; S.W., I.W.B., and X.H. wrote the paper.

This work was supported by National Institutes of Health Grant R01-EY-022443 and in part by the Office of the Director, National Institutes of Health Grant P51-0D-011106 to the Wisconsin National Primate Research Center, University of Wisconsin-Madison. This research was conducted at a facility constructed with support from Research Facilities Improvement Program Grants RR15459-01 and RR020141-01. We thank Jianbo Xiao for assistance on electrophysiological recordings and data analysis, Bryce Arseneau for technical support, Drs. Jennifer Coonen and Saverio Capuano for veterinary care, and Dr. Kevin Brunner for assistance with surgery. neurons in the visual cortex encode a single stimulus, how neurons encode multiple visual stimuli within their receptive fields (RFs) remains to be elucidated. Because visual perception depends critically on the integration and segregation of multiple visual stimuli (Braddick, 1993), understanding the neural representation of multiple stimuli is of significant importance.

\footnotetext{
*S.W. and I.W.B. contributed equally to this study.

The authors declare no competing financial interests.

Correspondence should be addressed to Xin Huang at Xin.Huang@wisc.edu.

https://doi.org/10.1523/JNEUROSCI.1950-19.2020

Copyright $\odot 2020$ the authors
} 
The middle temporal (MT) cortex is an extrastriate brain area that is important for visual motion processing (Britten, 2003; Born and Bradley, 2005; Park and Tadin, 2019). Neurons in area MT receive feedforward inputs from direction-selective neurons in V1 (Movshon and Newsome, 1996) and have RFs $\sim 10$ times larger in size than those of the neurons in the primary visual cortex (V1) at the same eccentricities (Gattass and Gross, 1981; Albright and Desimone, 1987). Previous studies have shown that neuronal responses in area MT elicited by multiple moving stimuli follow a sublinear summation of the responses elicited by the individual stimulus components (Snowden et al., 1991; Qian and Andersen, 1994; Ferrera and Lisberger, 1997; Recanzone et al., 1997; Britten and Heuer, 1999; Heuer and Britten, 2002; McDonald et al., 2014), consistent with a model of divisive normalization (Simoncelli and Heeger, 1998; Britten and Heuer, 1999; Carandini and Heeger, 2011).

Work in our laboratory has shown that the direction tuning curves of MT neurons to overlapping random-dot stimuli moving transparently in different directions can also be described as a weighted sum of the responses elicited by the individual stimulus components (Xiao et al., 2014; Xiao and Huang, 2015). When two stimulus components have different signal strengths in one feature domain, defined either by motion coherence or luminance contrast, MT neurons pool the stimulus component that has a stronger signal strength with greater weight (Xiao et al., 2014). The response bias in MT toward the stimulus component that has a stronger signal strength can be accounted for by a descriptive model of divisive normalization (Xiao et al., 2014), similar to the contrast normalization model used to describe neuronal responses in V1 (Carandini et al., 1997; Busse et al., 2009).

However, natural scenes contain multiple visual stimuli that often differ in more than one feature domain. For example, one stimulus may have a stronger signal strength in feature A but a weaker signal strength in feature B, whereas another stimulus may have a weaker signal strength in feature A but a stronger signal strength in feature B. In this case, it is unclear which stimulus has an overall stronger signal strength and, more generally, how visual stimuli with multiple competing features interact within neurons' RFs.

One possibility is that, for neurons in a given brain area, the overall signal strength of a visual stimulus is reflected in the evoked responses of a population of neurons in that area. Due to divisive normalization within that area, a neuron may weigh a visual stimulus more strongly if the population neural response elicited by that stimulus is greater than the population response elicited by a competing stimulus. Alternatively, how neurons in a given brain area weigh multiple competing stimuli may be the result of neural computations occurring in multiple stages along the hierarchical visual pathway and may not be explained by simply considering the population neural responses elicited by the individual stimulus components in the area of interest.

Here, we investigate the rule by which neurons in area MT encode multiple moving stimuli that compete in more than one feature domain. We found that MT responses to multiple stimuli changed drastically when the spatial arrangement of the visual stimuli was varied. Our results reveal how visual stimuli that differ in multiple feature domains interact within neurons' RFs and shed light on how the neuronal responses in a given cortical area are shaped by neural processing along the hierarchical visual pathway.

\section{Materials and Methods}

Two male adult rhesus monkeys (Macaca mulatta) were used in the neurophysiological experiments. Experimental protocols were approved by the Institutional Animal Care and Use Committee of the University of Wisconsin-Madison and conform to U.S. Department of Agriculture regulations and to the National Institutes of Health guidelines for the care and use of laboratory animals. Procedures for surgical preparation and electrophysiological recordings were routine and similar to those described previously (Xiao et al., 2015). A head post and a recording cylinder were implanted during sterile surgery with the animal under isoflurane anesthesia. For electrophysiological recordings from neurons in area MT, we took a vertical approach and used tungsten electrodes (1-3 M $\Omega$; FHC). We identified area MT by its characteristically large portion of directionally selective neurons, small RFs relative to those of neighboring medial superior temporal cortex (area MST), its location at the posterior bank of the superior temporal sulcus, and visual topography of the RFs (Gattass and Gross, 1981). Electrical signals were amplified and single units were identified with a real-time template-matching system and an offline spike sorter (Plexon). Eye position was monitored using a video-based eye tracker (EyeLink, SR Research) at a rate of $1000 \mathrm{~Hz}$.

Visual stimuli and experimental procedure. Stimulus presentation and data acquisition were controlled by a real-time data acquisition program, "Maestro" (https://sites.google.com/a/srscicomp.com/maestro/home). Visual stimuli were presented on a 25 -inch CRT monitor at a viewing distance of $63 \mathrm{~cm}$. Monitor resolution was $1024 \times 768$ pixels, with a refresh rate of $100 \mathrm{~Hz}$. Stimuli were generated by a Linux workstation using an OpenGL application that communicated with an experimental control computer. The luminance of the video monitor was measured with a photometer (LS-110, Minolta) and was gamma-corrected.

Visual stimuli were achromatic random-dot patches presented within a circular aperture with a diameter of $3^{\circ}$. Individual dots were squares of 2 pixels extending $0.08^{\circ}$ on each side, and each random-dot patch had a dot density of 2.7 dots/degree ${ }^{2}\left(\mathrm{deg}^{2}\right)$. The dots had a luminance of either 79 or $22 \mathrm{~cd} / \mathrm{m}^{2}$ and were presented on a uniform background with a luminance of $10 \mathrm{~cd} / \mathrm{m}^{2}$, which gives rise to a Michelson contrast of either 77.5 or $37.5 \%$. Random dots in each patch moved within the stationary aperture in a specified direction. The motion coherence of each random-dot patch was set to either 100 or $60 \%$. To generate a random-dot patch moving at $N \%$ of motion coherence (Newsome and Paré, 1988; Britten et al., 1992), N\% of the "signal" dots were selected to move coherently, whereas the rest of the dots, referred to as the "noise" dots, were repositioned randomly within the aperture. Random selections of the signal and noise dots occurred at each monitor frame. Therefore, a given dot would switch back and forth between a signal dot and a noise dot. The lifetime of each dot was as long as the motion duration.

In each experimental trial, the monkey maintained fixation within a $1^{\circ}$ $\times 1^{\circ}$ electronic window around a small fixation point. After a neuron was isolated, we first characterized its direction selectivity by interleaving trials of a $30^{\circ} \times 27^{\circ}$ random-dot patch, moving in different directions at a step of $45^{\circ}$ and at a speed of $10^{\circ} \%$. The direction selectivity and preferred direction (PD) were determined online using MATLAB (MathWorks). We then characterized the speed tuning of the neuron using a randomdot patch moving at different speeds $\left(1,2,4,8,16,32\right.$, or $\left.64^{\circ} / \mathrm{s}\right)$ in the PD of the neuron. Using a cubic spline, the preferred speed (PS) of the neuron was taken as the speed that evoked the highest firing rate in the fitted speed tuning curve. Next, we used a series of $5^{\circ} \times 5^{\circ}$ random-dot patches moving in the PD and at the PS of the neuron to map the RF of the neuron. The location of the patch was randomized, and the screen was tiled in $5^{\circ}$ steps. The RF map was interpolated at $0.5^{\circ}$ intervals, and the location giving rise to the highest firing rate was taken as the center of the RF.

In the main experiments, the visual stimuli appeared after the monkey maintained fixation for $200 \mathrm{~ms}$. To separate the neuronal responses to the stimulus motion from those due to the stimulus onset, the visual stimuli were first turned on and remained stationary for $200 \mathrm{~ms}$ before they started to move for $500 \mathrm{~ms}$. The visual stimuli were then turned off. The monkeys maintained fixation for an additional $200 \mathrm{~ms}$ after the stimulus offset. In some stimulus trials, two random-dot patches that moved in different directions, referred to as two stimulus components, were presented simultaneously. The direction separation between two stimulus components was fixed at $90^{\circ}$. We varied the vector average (VA) 

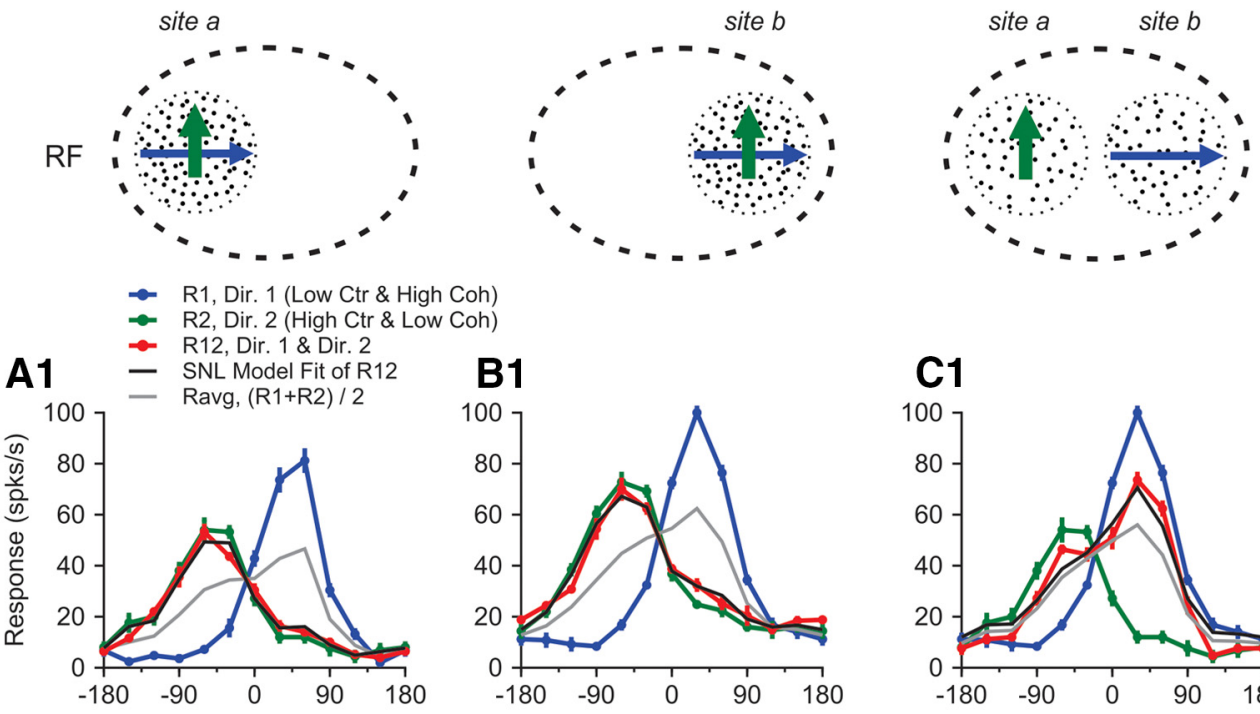

\section{B1}

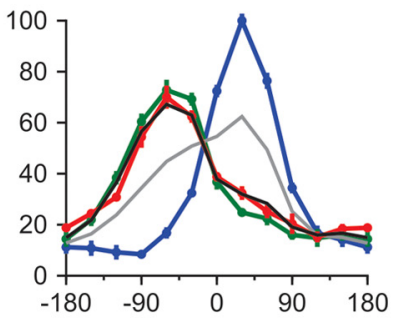

B2
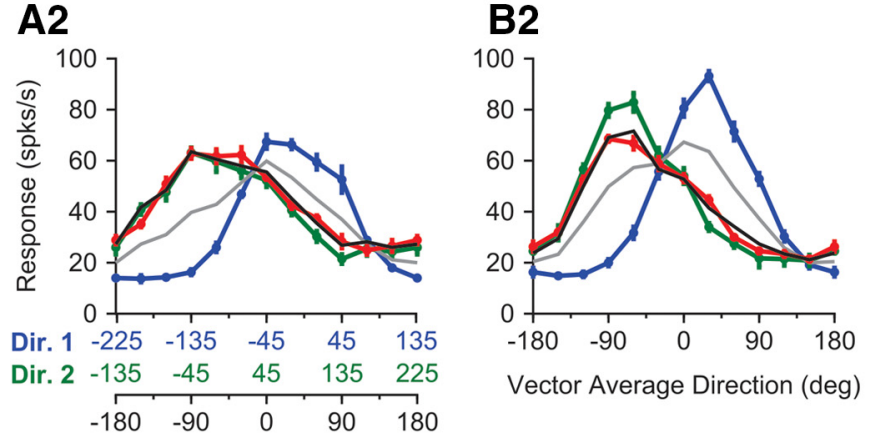

Vector Average Direction (deg)

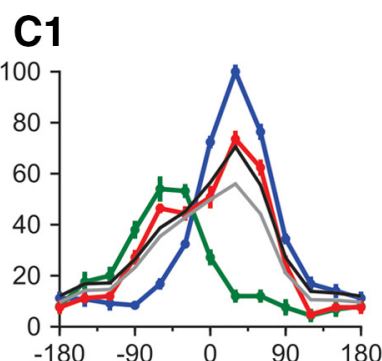

C2

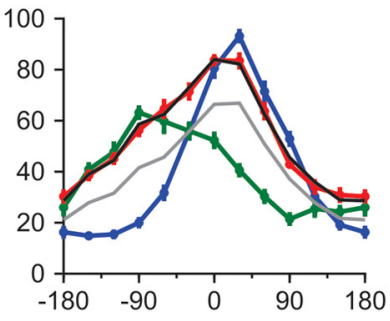

Vector Average Direction (deg)

Vector Average Direction (deg)

Figure 1. A1-C2, The response tuning curves of two example MT neurons to overlapping (A1-B2) and spatially separated stimuli (C1, $(2)$. Visual stimuli were achromatic random-dot patches moving in two directions separated by $90^{\circ}$. The low-contrast and high-coherence component (blue arrows) moved at the clockwise side of the two component directions, whereas the high-contrast and low-coherence component (green arrows) moved in the direction at the counter-clockwise side. The $x$-axis labeled in black indicates the vector average direction of the bidirectional stimuli. The $x$-axes labeled in blue and green (A2) indicate the direction of the low-contrast and high-coherence component (Dir. 1) and the direction of the high-contrast and low-coherence component (Dir. 2), respectively. The three $x$-axes are aligned such that the component directions shown in blue and green correspond to the directions of the two stimulus components at each vector average direction. A1-C1, Response tuning curves from one neuron. A2-C2, Response tuning curves from another neuron. The responses elicited by the bidirectional stimuli are shown in red (R12). The SNL model fits of R12 are shown in black. Error bars represent SEs.

direction of the bidirectional stimulus $\sim 360^{\circ}$ to characterize the response tuning curve. The two stimulus components were either overlapping in one of two locations (site a or b) within the RF or spatially separated within the RF, one centered at site a and the other at site $b$, with at least a $1^{\circ}$ gap between the borders of the two random-dot patches (illustrated in Fig. 1). In other trials, only one stimulus component was presented at either site a or site $b$, and the direction was varied to characterize the tuning curve to the stimulus component. For the majority of the experiments, the VA and component directions were varied in $15^{\circ}$ steps. In a small set of experiments, the directions were varied in $30^{\circ}$ steps. The trials presenting bidirectional stimuli and individual stimulus components were randomly interleaved.

In the first experiment, one random-dot patch, referred to as the "lowcontrast and high-coherence" component, had a luminance contrast of $37.5 \%$ and a motion coherence of $100 \%$. The other random-dot patch, referred to as the "high-contrast and low-coherence" component, had a luminance contrast of $77.5 \%$ and a motion coherence of $60 \%$. Both stimulus components moved at the same speed, which was set at the PS of the neuron if it was $<10 \%$, or at $10 \%$ if the PS was $\geq 10 \%$. Note that when a random-dot patch moved at $60 \%$ coherence in a given direction, the visual stimulus was different from a situation where $60 \%$ of the dots always moved coherently and the remaining $40 \%$ of dots always moved randomly. Because the random selection of signal and noise dots occurred at each monitor frame in our stimuli, a noise dot at one frame may turn into a signal dot in the next frame and move in the coherent direc- tion. Perceptually, it is difficult to segregate the noise dots from the signal dots of the same stimulus component. The noise dots of the highcontrast and low-coherence component are not an independent entity and do not appear to interfere with the coherence of the low-contrast and high-coherence component perceptually.

In the second experiment, we set the motion coherence of both random-dot patches to $100 \%$ but used different speeds for the two stimulus components. One random-dot patch, referred to as the "low contrast and faster speed" component, had a luminance contrast of $37.5 \%$ and moved at $10 \%$ s. The other random-dot patch, referred to as the high-contrast and slower-speed" component, had a luminance contrast of $77.5 \%$ and moved at $2.5 \%$ s.

Experimental design and statistical analysis. Response firing rate was calculated during the period of $500 \mathrm{~ms}$ stimulus motion and averaged across repeated trials. We fitted the raw direction tuning curves for the bidirectional stimuli and the individual stimulus components using splines at a resolution of $1^{\circ}$. We then rotated the spline-fitted tuning curve to the bidirectional stimuli so that the VA direction of $0^{\circ}$ was aligned with the PD of each neuron. In the first experiment, the responses of each neuron to the bidirectional stimuli and individual stimulus components were normalized by the maximum response to the low-contrast and high-coherence component. In the second experiment, the responses of each neuron were normalized by the maximum response to the faster speed component. We averaged the rotated and normalized tuning curves across neurons to obtain population-averaged tuning curves. 
Table 1. Model parameters for V1 and MT neurons

\begin{tabular}{lll}
\hline Model parameters & V1 stage & MT stage \\
\hline RF size (pixels) & 15 (simple cell) & 211 \\
SD of Gaussian RF profile (pixels) & $\begin{array}{l}25 \text { (complex cell) } \\
\end{array}$ & \\
& cells) & 53 \\
& 7 (complex cell) & \\
$\sigma^{2}\left(C_{50}\right)$ & 0.0016 & 0.000049 \\
Size of normalization pool $m$ (pixels) & 38 & 153 \\
SD $D_{\text {norm }}$ (pixels) & 5 & 52 \\
Baseline activity & 0 & 0.1 \\
\hline
\end{tabular}

To quantify the relationship between the responses elicited by the bidirectional stimuli and those elicited by the individual stimulus components, we fitted the direction tuning curves using a summation plus nonlinear interaction (SNL) model (Eq. 1), which has been shown to provide a better fit of MT responses elicited by bidirectional stimuli than a linear weighted summation model (Xiao et al., 2014):

$$
R_{\text {pred }}\left(\theta_{1}, \theta_{2}\right)=w_{1} R_{1}\left(\theta_{1}\right)+w_{2} R_{2}\left(\theta_{2}\right)+b R_{1}\left(\theta_{1}\right) R_{2}\left(\theta_{2}\right),
$$

where $R_{\text {pred }}$ is the response to the bidirectional stimuli predicted by the model; $\theta_{1}$ and $\theta_{2}$ are the two component directions; $R_{1}$ and $R_{2}$ are the measured component responses elicited by the two stimulus components when presented alone; $w_{1}$ and $w_{2}$ are the response weights for $R_{1}$ and $R_{2}$, respectively; and $b$ is the coefficient of multiplicative interaction between the component responses. To determine whether the response elicited by the bidirectional stimuli showed a significant bias toward one of the two stimulus components, we compared the response weights, $w_{1}$ and $w_{2}$ using either a paired $t$ test or a Wilcoxon signed-rank test.

We also fitted the response tuning curves to the bidirectional stimuli using a few variants of a divisive normalization model (Carandini and Heeger, 2011; see Results). The model fits were obtained using the constrained minimization tool "fmincon" (MATLAB) to minimize the sum of squared error.

To evaluate the goodness of fit of a model for the response tuning curve to the bidirectional stimuli, we calculated the percentage of variance (PV) accounted for by the model as follows:

$$
P V=100 \times\left(1-\frac{S S E}{S S T}\right),
$$

where SSE is the sum of squared errors between the model fit and the neuronal data, and SST is the sum of squared differences between the data and the mean of the data (Morgan et al., 2008).

V1-MT model. We adapted a computational model proposed by Simoncelli and Heeger (1998; http://www.cns.nyu.edu/ lcv/MTmodel/) to reconstruct our visual stimuli and to simulate the neuronal response tuning to the bidirectional stimuli that were either overlapping or spatially separated. The model contained several consecutive stages, which can be interpreted as V1 simple, V1 complex, and MT (Simoncelli and Heeger, 1998; Rust et al., 2006). Based on the dimensions of the video monitor and the viewing distance in our neurophysiological experiments, $1^{\circ}$ of visual angle corresponds to 21 pixels. The random-dot patch in our model simulations had a circular aperture with a diameter of 63 pixels (i.e., $3^{\circ}$ ) and the same dot density as used in our experiments. Each dot had a size of $2 \times 2$ pixels.

We set the RFs of model neurons by Gaussian convolutional filters (Table 1). We estimated the size of the RF for each neuron type by summing the lengths of the incorporated filters. For the spatially separated stimuli, we set a blank gap between the two stimulus components as the RF size of the V1 complex neuron, which is $1.2^{\circ}$, to ensure that no V1 neuron would be driven by both stimulus components. We generated direction-selective neuron populations that approximately tiled a sphere in the frequency domain. We tuned the contrast response functions by adjusting $C_{50}$ values for V1 and MT neurons. These $C_{50}$ values were represented in the model as $\sigma^{2}$ in the normalization equation (Eq. 3), which was applied to both V1 complex cell and MT stages of the model (adapted from Simoncelli and Heeger, 1998; Rust et al., 2006):

$$
R_{n}^{\prime}(t)=\frac{\left\lfloor R_{n}(t)\right\rfloor^{2}}{K \sum_{m} w_{m} \cdot\left\lfloor R_{m}(t)\right\rfloor^{2}+\sigma^{2}},
$$

where $R_{n}(t)$ represents the linear filter response of the $n$th neuron; $R_{n}^{\prime}(t)$ represents the normalized response of either the V1 complex cell or MT neuron; $L\rfloor$ denotes half-wave rectification; $K$ represents the strength of normalization, which was set as $1-\sigma^{2} ; m$ represents the normalization pool of the $n$th neuron; $w$ represents the Gaussian spatial weighting profile of the normalization pool, with an $\mathrm{SD}$ of $\mathrm{SD}_{\text {norm }}$. The model parameters for V1 and MT stages are defined in Table 1 . We fitted the model contrast response functions to neural data from V1 and MT as described by Sclar et al. (1990). Similarly, we tuned coherence responses by varying the spatial scale of the normalization pool $(m)$, the weighting profile within the pool $(w)$, and the size of the V1 linear RF. The MT coherence response function was fitted to data replotted from Figure $1 C$ in Britten and Newsome (1998). We are not aware of published neural data on V1 coherence response function. Therefore, the parameters for V1 model neurons were varied to simulate our MT responses to bidirectional stimuli without a constraint on V1 coherence response function. The same model parameters were used for the overlapping and spatially separated conditions.

We explored several variants of the model architecture. The model parameters were fitted after each architectural manipulation. The following changes enabled the model to better capture the trends of the stimulus competition found in our neural data. First, we used area-normalized Gaussian functions to set the weights for the spatial pooling and local population normalization. Second, multiple frequency scales for V1 simple cells were computed by tripling the SD of the underlying third-order derivative Gaussian, similar to the doubling suggested by Simoncelli and Heeger (1998) - this change was made after spectral analysis of stimuli showed that a wider range of scales was necessary to capture motion at lower coherence. Third, V1 afferent weights were not adjusted to zero mean, allowing MT neurons to have variable proportions of positive and negative inputs. Finally and importantly, rectification and static nonlinearity were applied to the MT stage after spatial pooling and before normalization, which is physiologically plausible and provides a better fit of our neural data.

\section{Results}

We asked the question of how neurons in extrastriate area MT represent multiple visual stimuli that compete in more than one feature domain. To address this question, we conducted neurophysiological experiments and computer simulations. We recorded electrophysiological data from isolated single neurons in area MT of two macaque monkeys while they performed a fixation task. Visual stimuli were two random-dot patches moving simultaneously in different directions within the RFs. In the first experiment, we used luminance contrast and motion coherence as two competing features. One stimulus had high contrast but moved with low coherence, whereas the other stimulus had low contrast but moved with high coherence (see Materials and Methods). We manipulated the spatial arrangement of the visual stimuli to investigate the contributions of earlier visual areas and area MT in mediating the competition between multiple stimuli. In a second experiment, we used luminance contrast and motion speed as two competing features. We first present the results from the neurophysiological experiments and then computer simulations.

\section{Neurophysiological experiments}

We measured the direction tuning curves of MT neurons in response to two stimuli that had competing visual features and moved simultaneously in different directions. Our dataset includes recordings from $76 \mathrm{MT}$ neurons, 43 from monkey $\mathrm{G}$ and 

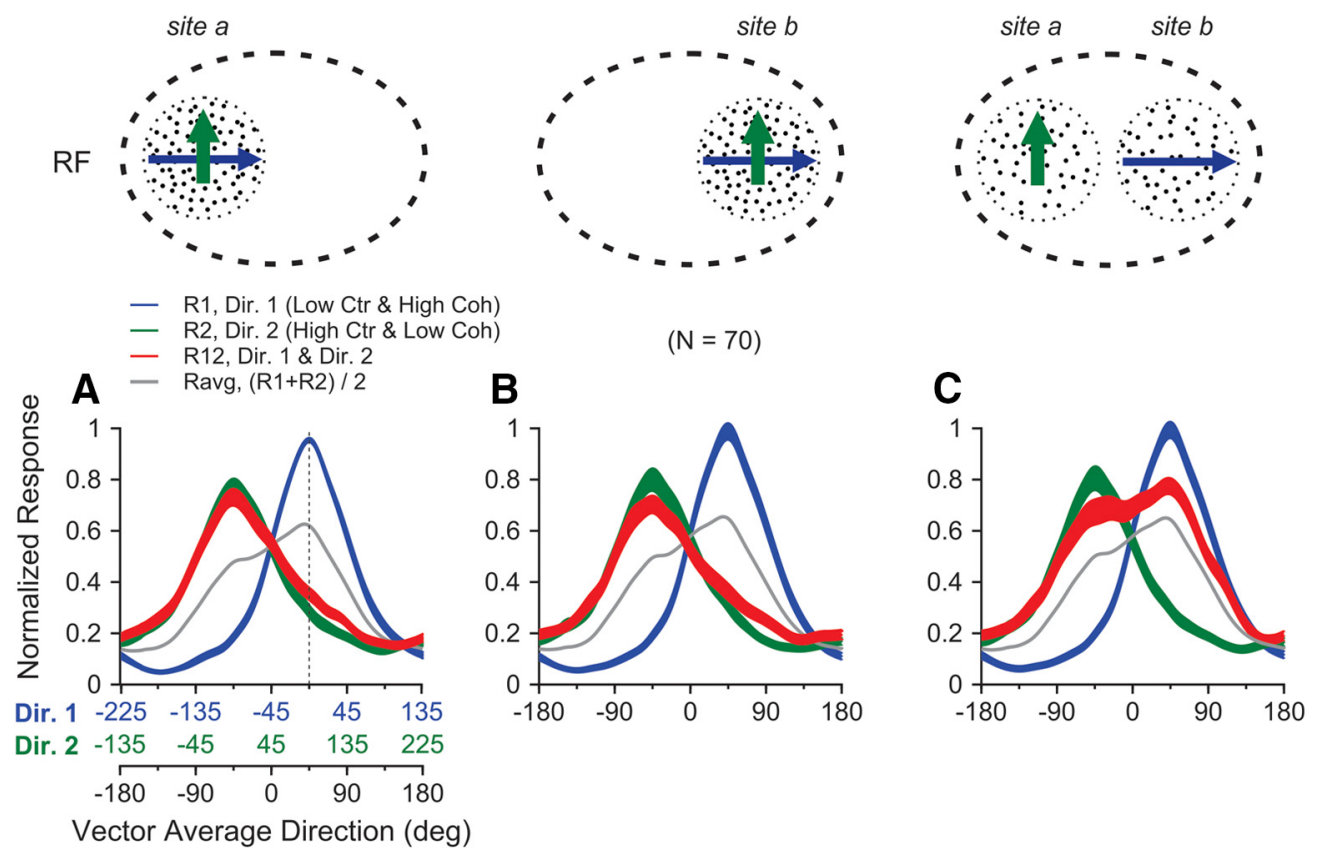

Figure 2. Population-averaged tuning curves to the bidirectional stimuli (red) and the unidirectional stimulus components (blue and green). $A$, The vector average direction of the bidirectional stimuli and the directions of individual stimulus components are labeled in the corresponding $x$-axes, following the same convention as in Figure 1 . The direction of $0^{\circ}$ was aligned with the $P D$ of each neuron before the tuning curves were averaged across neurons. $\boldsymbol{A}-\boldsymbol{C}$, The stimulus components were overlapping at site $a(\boldsymbol{A})$ or site $b(\boldsymbol{B})$ or were spatially separated $(\boldsymbol{C})$ within the RFs. The width of each tuning curve represents the $S E$. The average of the responses to the two stimulus components is shown in gray.

33 from monkey B. We set the angular separation between the motion directions of two individual stimuli, referred to as the stimulus components, at $90^{\circ}$ and varied the VA direction of the stimuli. In the first experiment, one stimulus component had a low contrast of $37.5 \%$ and moved at a high motion coherence of $100 \%$. The other component had a high contrast of $77.5 \%$ and moved at a low coherence of $60 \%$. Figure 1 shows the direction tuning curves of two representative neurons. The red curve shows the neuronal response elicited when both stimulus components were present, as a function of the VA direction of the two stimulus components. The green and blue curves show the neuronal responses elicited by the individual stimulus components when presented alone. The tuning curves of the component responses are arranged such that, at each VA direction, the data points on the green and blue curves correspond to the responses elicited by the individual stimulus components of that VA direction (note the color-coded abscissas for the component directions in Fig. 1A2).

For the two example neurons, the peak response of the direction tuning curve to the low-contrast and high-coherence component alone (shown in blue) was greater than that of the high-contrast and low-coherence component (shown in green; Fig. 1). This is expected because MT neurons are sensitive to motion coherence within a large coherence range (Britten et al., 1993), whereas their contrast response function saturates at a low luminance contrast (Sclar et al., 1990). Consequently, the average of the response tuning curves to the two stimulus components (shown in gray) was biased toward the low-contrast and highcoherence component. Surprisingly, we found that when the two stimulus components were overlapping, the neuronal responses elicited by the bidirectional stimuli were strongly biased toward the high-contrast and low-coherence component (Fig. 1A1,A2). This response bias was robust and occurred when we placed the overlapping stimuli at a different site within the RF (Fig. 1B1,B2).
Two overlapping visual stimuli could stimulate not only the RFs of single MT neurons but also the RFs of single V1 neurons. The response bias toward the high-contrast and low-coherence component may be caused by the neural processes within area MT or, alternatively, inherited from earlier visual areas, such as V1. To determine the contribution of earlier visual areas to the response bias, we placed two stimulus components at different locations within the RF of a given MT neuron. The two stimulus components were separated by a gap of at least $1^{\circ}$ (illustrated in Fig. 1C1,C2). With this spatial arrangement, the RF of a single V1 neuron could only be stimulated by one of the two stimulus components, whereas the RF of an MT neuron could still be stimulated by both components. We found that the response tuning to the bidirectional stimuli changed drastically when stimulus components were spatially separated. MT responses elicited by the bidirectional stimuli no longer showed a bias toward the high-contrast and lowcoherence component but approximately followed a scaled average of the component responses (Fig. 1C1,C2).

Figure 2 shows the tuning curves averaged across 70 MT neurons. The population-averaged response elicited by the lowcontrast and high-coherence component moving in the PD of each neuron, aligned to $0^{\circ}$, was significantly greater than that elicited by the high-contrast and low-coherence component moving in the PD (one-tailed paired $t$ test, $p=4.1 \times 10^{-7}$ ). However, when the two stimuli were overlapping, the population response elicited by the bidirectional stimuli was almost completely biased toward the weaker high-contrast and low-coherence component, regardless of the spatial location within the RF (Fig. 2A,B). The bias toward the high-contrast and low-coherence component at a given VA direction was in a manner of "higher contrast take all." For example, at a VA direction of $45^{\circ}$ where the lowcontrast and high-coherence component moved in the $\mathrm{PD}\left(0^{\circ}\right)$ and the high-contrast and low-coherence component moved in a $90^{\circ}$ direction (indicated by a dotted line in Fig. $2 \mathrm{~A}$ ), the bidirec- 
tional response closely followed the much weaker response elicited by the high-contrast and low-coherence component. When the two stimulus components were spatially separated within the $\mathrm{RF}$, the strong bias toward the high-contrast and low-coherence component was abolished (Fig. 2C). The population response to the bidirectional stimuli now showed approximately equal weighting of the responses elicited by the individual stimulus components.

The SNL model (see Eq. 1 in Materials and Methods) provided an excellent fit of the MT responses elicited by the bidirectional stimuli, illustrated by the black curves in Figure 1. Across our neuron population, the model fit accounted for $83 \%$ of the response variance on average (see Materials and Methods). Figure 3 compares the response weights for the two stimulus components obtained from the SNL model fits. In the overlapping condition, the mean response weight $\left(w_{2}\right)$ for the high-contrast and lowcoherence component was significantly greater than weight $w_{1}$ for the low-contrast and high-coherence component (one-tailed paired $t$ test, $p=1.9 \times 10^{-45}$ for site a, $p=2.5 \times 10^{-28}$ for site b; Fig. $3 A$ ). Nearly all data points, each representing the result from one neuron, were below the unity line. The mean response weight for the high-contrast and low-coherence component was 0.97 (SD $=0.24$ ), whereas the mean weight for the low-contrast and highcoherence component was $0.23(\mathrm{SD}=0.25)$, indicating a dominant effect of the high-contrast and low-coherence component in determining the neuronal response to the bidirectional stimuli.

When the two stimulus components were spatially separated within the RF, the response weights changed significantly, becoming symmetrically distributed relative to the unity line (Fig. $3 B$ ). The spread of weights in the spatially separated condition is larger than that in the overlapping condition. The mean weight for the high-contrast and low-coherence component decreased to 0.66 ( $\mathrm{SD}=0.32$ ), whereas the mean weight for the lowcontrast and high-coherence component increased to 0.68 (SD $=$ 0.43 ). The mean weights for the two components were no longer different (paired $t$ test, $p=0.8$ ) but were significantly $>0.5$ of response averaging ( $t$ test, $p<0.001)$.

To quantify the response bias toward an individual stimulus component, we calculated a bias index (BI):

$$
B I=\left(w_{2}-w_{1}\right) /\left(w_{2}+w_{1}\right) .
$$

A positive value of the index indicates a bias toward the highcontrast and low-coherence component. Figure $3 C$ shows how this bias index changes with the spatial arrangement of the visual stimuli. In the overlapping condition, the mean BI is 0.71 (median $=0.70, \mathrm{SD}=0.23$ ), which is significantly $>0$ (one-tailed $t$ test, $\left.p=7.5 \times 10^{-35}\right)$. In the spatially separated condition, the mean $\mathrm{BI}$ is -0.05 (median $=-0.01, \mathrm{SD}=0.95$ ), which is not significantly different from $0(p=0.7)$. The mean BI obtained in the overlapping condition is significantly greater than that in the spatially separated condition (one-tailed paired $t$ test, $p=4.7 \times$ $10^{-9}$ ), indicating a change of the response bias when the spatial arrangement of the visual stimuli is altered.

We previously found that the tuning curves of some MT neurons to overlapping bidirectional stimuli can show a directional "side bias" toward one of the two direction components (Xiao and Huang, 2015). A subgroup of neurons prefer the stimulus component on the clockwise side of two motion directions, whereas another group prefers the component direction on the counterclockwise side. These response biases can occur even when both stimulus components have the same contrast and coherence. In the experiment shown in Figures 1, 2, and 3, the high-contrast and low-coherence component
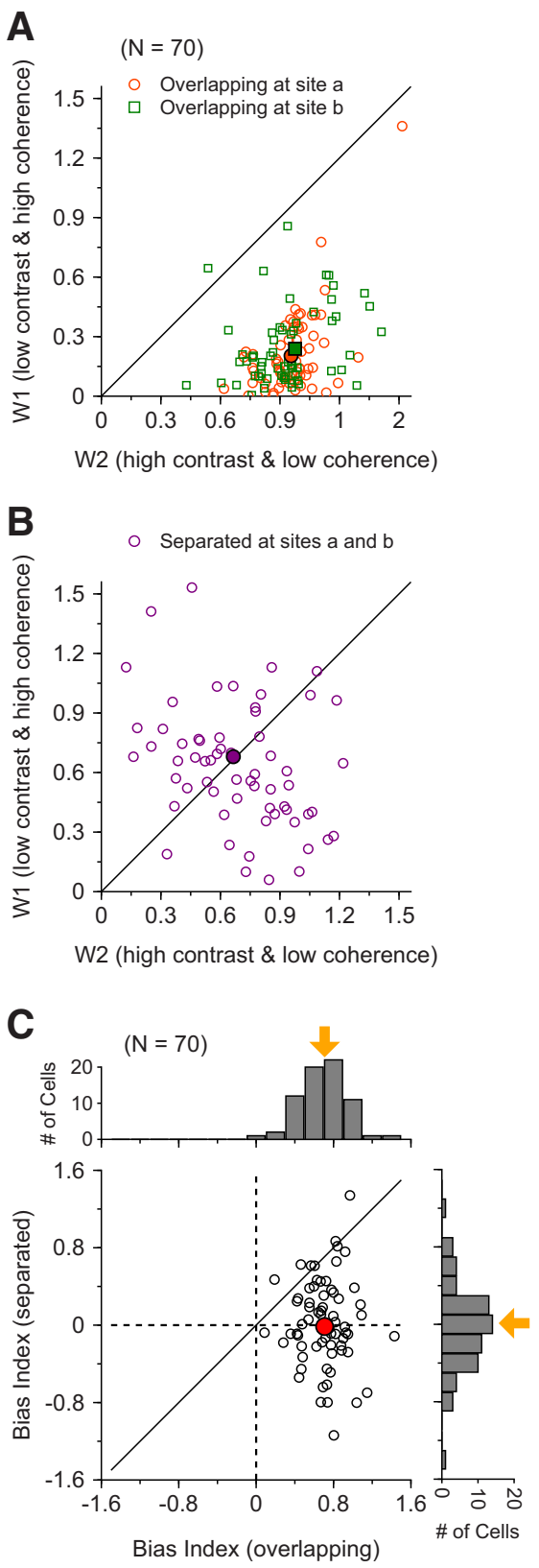

Figure 3. The effect of the spatial arrangement of the bidirectional stimuli on the response weights for the stimulus components. Each dot represents the result from one neuron. $\boldsymbol{A}, \boldsymbol{B}$, Comparison of the response weights for the low-contrast and high-coherence component (ordinate) with the high-contrast and low-coherence component (abscissa) under the overlapping $(\boldsymbol{A})$ and the spatially separated $(\boldsymbol{B})$ conditions. $\boldsymbol{C}$, Comparison of the bias indices between the spatially separated (ordinate) and overlapping (abscissa) conditions. The histograms ( $\boldsymbol{C}$ ) show the distributions of the bias index for the overlapping (top) and spatially separated (right) conditions.

always moved in the counterclockwise side direction (Fig. 2A,B). Could the strong bias toward the high-contrast and low-coherence component in the overlapping condition be due to a biased neuron sample that happened to have a strong bias toward the direction component on the counterclockwise side? To address this concern, we arranged the direction components differently.

Figure 4, $A$ and $B$, shows the average direction tuning curves of 15 MT neurons when the direction of the high-contrast and lowcoherence component was placed on the counterclockwise side under the overlapping and spatially separated conditions, as in Figure 2. When the high-contrast and low-coherence component 


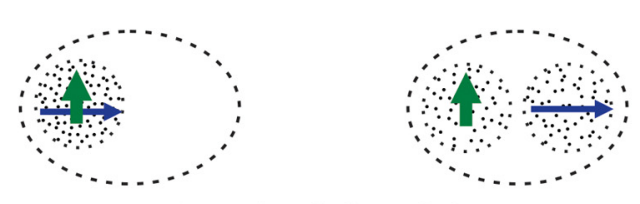

- R1, Dir. 1 (Low Ctr \& High Coh)

- R2, Dir. 2 (High Ctr \& Low Coh)

- R12, Dir. 1 \& Dir. 2

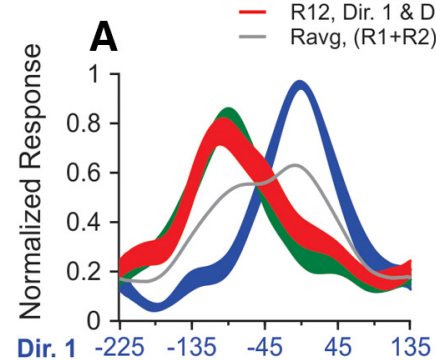

B $\quad(\mathrm{N}=15)$

Dir. 2 -135 $-45 \quad 45 \quad 135 \quad 225$

$\begin{array}{lllll}-180 & -90 & 0 & 90 & 180\end{array}$

Vector Average Direction (deg)
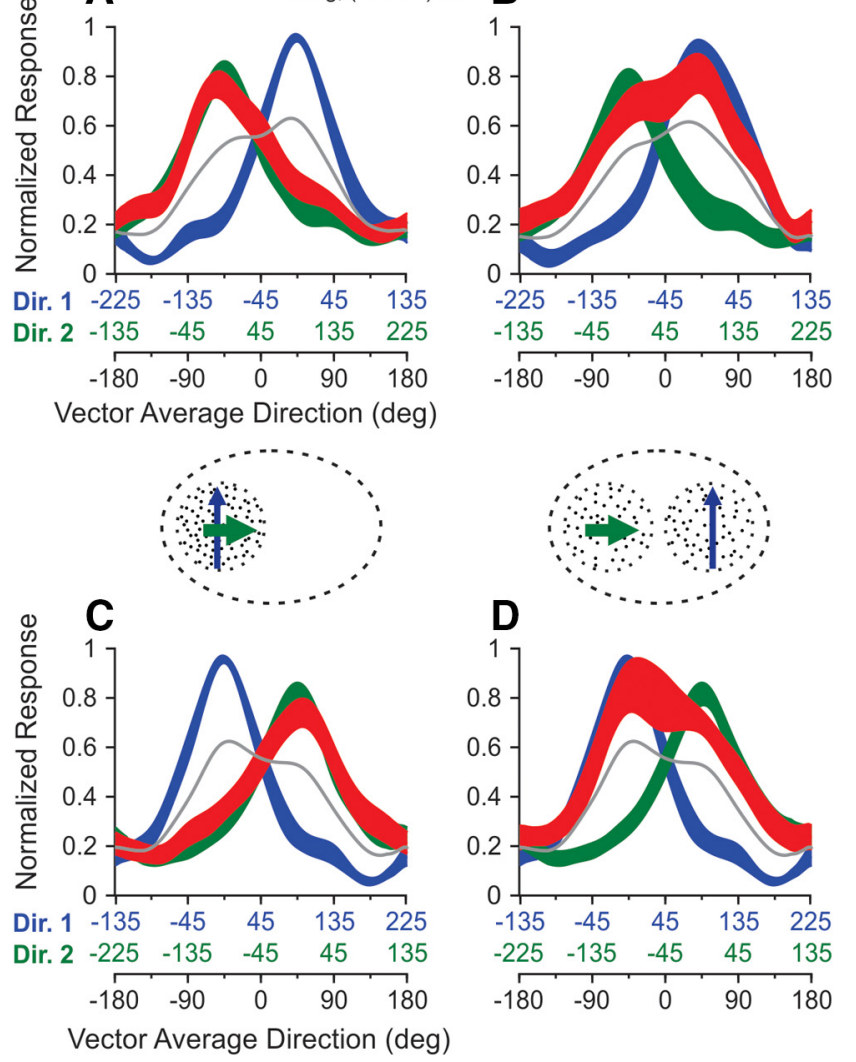

Figure 4. Control for the directional arrangement of the two stimulus components. $\boldsymbol{A}, \boldsymbol{B}$, Response tuning curves averaged across 15 MT neurons to the bidirectional stimuli and the stimulus components when the direction of the high-contrast and low-coherence component was placed on the counterclockwise side of the two component directions, as in Figures 1 and 2. $C, D$, Response tuning curves averaged across the same 15 neurons when the direction of the high-contrast and low-coherence component was placed on the clockwise side of the two component directions. $\boldsymbol{A}, \boldsymbol{C}, 0$ verlapping condition. $\boldsymbol{B}, \boldsymbol{D}$, Spatially separated condition. Notice the switch of the values in the $x$-axes of the component directions, shown in blue and green, between $\boldsymbol{A}$ and $\boldsymbol{C}$, as well as between $\boldsymbol{B}$ and $\boldsymbol{D}$.

was placed on the clockwise side of the two component directions, the responses of the same 15 neurons to the bidirectional stimuli still showed a strong bias toward the high-contrast and low-coherence component under the overlapping condition (Fig. 4C) and showed approximately equal weighting of the two components under the spatially separated condition (Fig. 4D). Placing the high-contrast and low-coherence component on the clockwise or counterclockwise side of the two component directions had no effect on the response bias, as measured by the bias index under the overlapping and spatially separated conditions (Wilcoxon rank-sum test, $p=0.6$ ).

To shed light on the neural mechanisms underlying the response bias, we examined the time course of the neuronal responses in the overlapping and spatially separated conditions. Figure 5 shows the peristimulus time histograms (PSTHs) calculated using a $10 \mathrm{~ms}$ time bin when either the high-contrast and low-coherence component or the low-contrast and high-coherence component moved in the PD. When stimuli were overlapping, as soon as MT neurons started to respond to the onset of the static stimuli (see Materials and Methods), the response elicited by both the high-contrast and low-contrast components (the red curves in Fig. $5 A, B$ ) already closely followed the response elicited by the highcontrast component alone (the green curves in Fig. 5A,B), even before the onset of the stimulus motion.

As soon as the neuronal response to stimulus motion started, the neuronal response to the bidirectional stimuli followed the response elicited by the high-contrast and low-coherence component throughout the motion period, regardless of whether the component moved in the PD and elicited a strong response (Fig. $5 A$ ) or $90^{\circ}$ away from the PD and elicited a weak response (Fig. $5 B)$. Because the strong bias toward the high-contrast and lowcoherence component in the overlapping condition occurred at the very beginning of the response onset, it is unlikely that the bias was due to selective attention because attention modulation is delayed relative to the neural response onset (Wannig et al., 2007; Lee and Maunsell, 2010; Ni et al., 2012).

When stimuli were spatially separated, MT neurons also followed the high-contrast and low-coherence component in response to the onset of the static stimuli (Fig. $5 C, D$ ). After the motion onset, when the high-contrast and low-coherence component moved in the PD, the motion response elicited by the bidirectional stimuli initially followed the high-contrast and lowcoherence component for $\sim 30 \mathrm{~ms}$ and was then "pulled down" by the non-PD component (Fig. $5 C$, arrow). When the highcontrast and low-coherence component moved in the non-PD, the motion response elicited by the bidirectional stimuli followed the high-contrast and low-coherence component for $\sim 10 \mathrm{~ms}$ after the onset of the motion response to the PD component and was then "pulled up" by the PD component (Fig. 5D, arrow). These results suggest that response normalization under the spatially separated condition takes $\sim 10-30$ ms to occur.

When two stimulus components overlap, the random dots from each component constitute only half of the total number of dots of the two moving surfaces. Could the strong response bias toward the high-contrast and low-coherence component be due to a reduction of the motion coherence of the low-contrast and high-coherence component when the stimuli overlapped? We think this is an unlikely explanation because overlapping reduces the percentage of the signal dots relative to the total number of dots for both stimulus components. In addition, our stimuli moved in two directions separated by $90^{\circ}$. Human observers can reliably segregate the two stimulus components at this angle separation, and the low-contrast and high-coherence component still appears to move coherently. Overlapping does not change the relative coherence levels nor the perceived coherence of the two stimulus components. When overlapping random-dot stimuli have the same luminance contrast but move at different motion coherences, macaque MT response to both stimulus components is biased toward the high-coherence component (Xiao et al., 2014), indicating that stimulus overlapping does not prevent the response bias toward the high-coherence component given equal contrast.

To determine whether the dominance by the high-contrast component on MT responses elicited by overlapping stimuli occurs only when luminance contrast and motion coherence compete with each other, we conducted a second experiment using visual stimuli that differ in luminance contrast and motion speed. We previously found that when two overlapping random-dot patches moved in the same direction at different speeds, within a range of low to intermediate speeds, the responses of MT neurons 

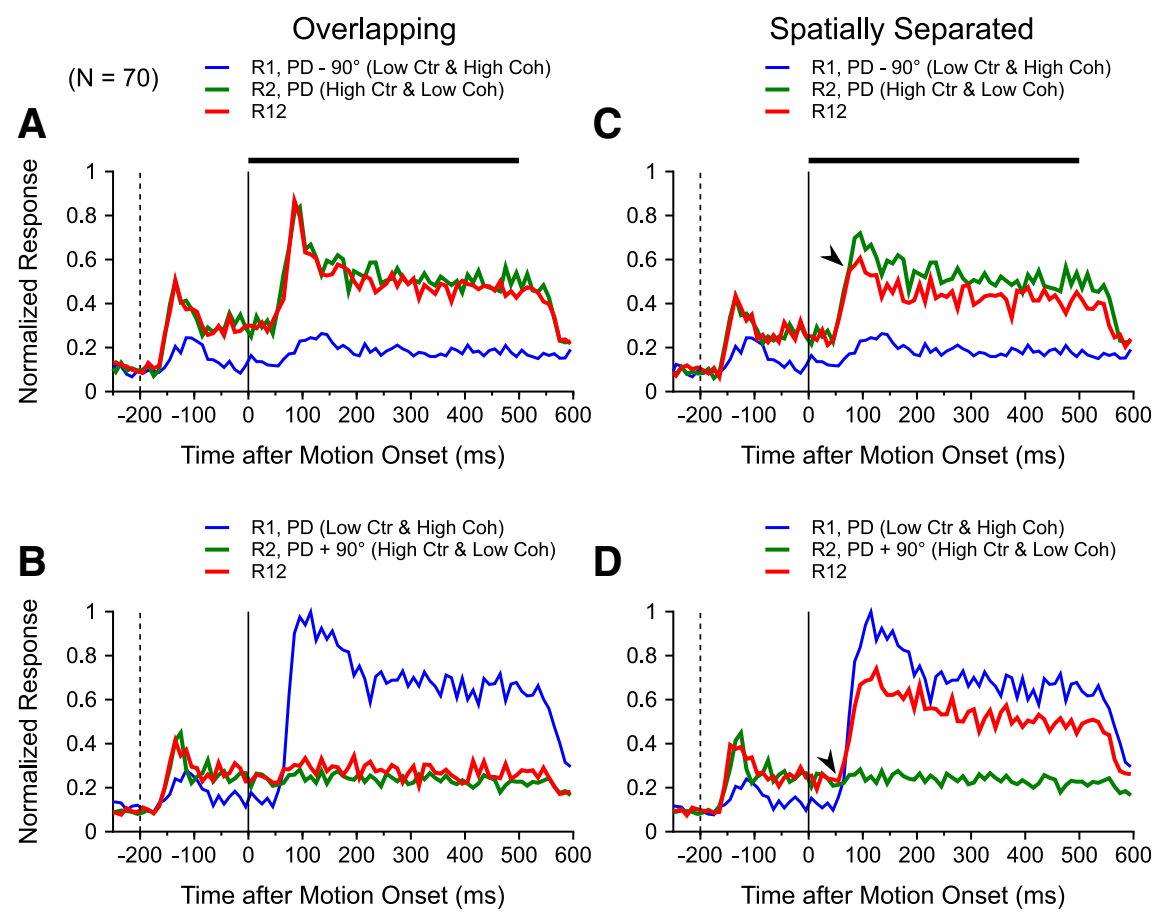

Figure 5. Time course of the neuronal responses to the bidirectional stimuli and the stimulus components. PSTHs were calculated using a $10 \mathrm{~ms}$ time bin and averaged across 70 neurons. $\boldsymbol{A}, \boldsymbol{B}$, The two stimulus components overlapped (at site a) within the RF. $C, D$, The two stimulus components were spatially separated within the RF. The dashed vertical lines at -200 ms indicate the onset of the static stimuli. The solid vertical lines at time 0 indicate motion onset. $\boldsymbol{A}, \boldsymbol{C}$, The solid horizontal bars indicate the stimulus motion period. The high-contrast and low-coherence component moved in the PD in $\boldsymbol{A}$ and $\boldsymbol{C}$ and in a non-PD in $\boldsymbol{B}$ and $\boldsymbol{D}$.

elicited by the bispeed stimuli were biased toward the faster speed component (Huang et al., unpublished observations). Motivated by this finding, we used motion speed to compete with luminance contrast. As in the main experiment, the visual stimuli contained two random-dot patches moving in two directions separated by $90^{\circ}$, and we varied the VA direction to measure the direction tuning curves. One stimulus component had a high luminance contrast of $77.5 \%$ and moved at a slower speed of $2.5 \%$. The other stimulus component had a low luminance contrast of $37.5 \%$ and moved at a faster speed of $10 \%$. Both stimulus components moved at $100 \%$ coherence and were either overlapping or spatially separated within the RF of a given MT neuron, as in the first experiment. We also measured the direction tuning curves when the two stimulus components both had high luminance contrast $(77.5 \%)$ and moved at $2.5 \%$ s and $10 \%$, respectively, at $100 \%$ coherence.

We recorded from 13 MT neurons using these visual stimuli. Figure 6 shows the population-averaged tuning curves. When both stimulus components had high contrast, the peak response elicited by the faster $\left(10^{\circ} \mathrm{s}\right)$ stimulus component moving in the PD (i.e., $0^{\circ}$ ) was greater than that elicited by the slower $\left(2.5^{\circ} / \mathrm{s}\right)$ component moving in the $\mathrm{PD}$. The component responses are shown in green and purple in Figure $6 \mathrm{~A}$. When the two stimulus components were overlapping, the tuning curve elicited by both stimulus components (shown in red) is biased toward the faster stimulus component, more than what is predicted by the average of the component responses (shown in gray; Fig. 6A). We fitted the direction tuning curves using the SNL model for each neuron (Eq. 1). The median response weight obtained by the model fit for the faster stimulus component (0.88) was significantly greater than the median weight $(0.41)$ for the slower component (Wilcoxon signed-rank test, $p=7.3 \times$ $\left.10^{-4}\right)$. This result extended our previous finding of the response bias toward the faster stimulus component for stimuli moving in the same direction (unpublished results) to stimuli moving in different directions.

When the overlapping stimuli moving at different speeds had different luminance contrasts, the responses elicited by both stimulus components showed a strong bias toward the high-contrast and slower-speed component, even though the peak response to this component alone was significantly weaker than that to the low-contrast and faster-speed component (Fig. 6B). We found the same result when the two stimulus components overlapped at a different site within the RF (Fig. 6C). Under the overlapping condition, the median response weight for the high-contrast and slower-speed component was 0.81 , which was significantly greater than the median weight for the low-contrast and faster-speed component (0.17; Wilcoxon signed-rank test, $p=$ $\left.2.4 \times 10^{-4}\right)$. Separating the two stimulus components spatially within the RF abolished the bias toward the high-contrast and slower-speed component (Fig. 6D). As the spatial arrangement of the stimulus components changed from overlapping to spatially separated, the median bias index (Eq. 4) decreased significantly from $0.65(\mathrm{SD}=0.21)$ to $-0.08(\mathrm{SD}=0.37$; Wilcoxon signed-rank test, $p=0.0012$ ). The values of the bias indices under the overlapping and spatially separated conditions were consistent with the bias indices calculated when visual stimuli competed between contrast and motion coherence. These results confirmed that luminance contrast has a dominant effect on MT responses elicited by overlapping stimuli, which is not unique to the competition between contrast and motion coherence. The spatial arrangement of visual stimuli can substantially change the competition between multiple stimuli within the RF.

Fitting response tuning curve using the normalization model Previous studies have shown that neuronal responses elicited by multiple stimuli in many brain areas can be described by a divisive normalization model (Carandini and Heeger, 2011). We asked whether our results could also be accounted for by response normalization. We first fitted the data using the following equation:

$$
R_{\text {pred }}\left(\theta_{1}, \theta_{2}\right)=\frac{S_{1}^{n}}{S_{1}^{n}+S_{2}^{n}+\sigma} R_{1}\left(\theta_{1}\right)+\frac{S_{2}^{n}}{S_{1}^{n}+S_{2}^{n}+\sigma} R_{2}\left(\theta_{2}\right)+c,
$$

where $R_{1}$ and $R_{2}$ are the evoked direction tuning curves to the two stimulus components 1 and 2 , respectively; $\theta_{1}$ and $\theta_{2}$ are the component directions; $S_{1}$ and $S_{2}$ represent the signal strengths of the low-contrast and high-coherence component and the highcontrast and low-coherence component, respectively (see definition below); $R_{\text {pred }}$ is the model-predicted response elicited by both stimulus components presented simultaneously; and $n, \sigma$, 
and $c$ are model parameters with the constraints of $n \geq 1$ and $c>0$. Equations of a similar form have been used previously to describe normalization involving contrast, in which case the signal strength is simply the luminance contrast (Carandini et al., 1997; Busse et al., 2009; Xiao et al., 2014; Bao and Tsao, 2018). Because our visual stimuli competed in more than one feature domain, it was not obvious which stimulus component had an overall stronger signal strength. Because the brain has to make an inference of the signal strength based on the elicited neural responses, we assumed that the signal strength of a stimulus component, in the "eye" of MT neurons, is reflected in the neural responses elicited by that stimulus component moving in a fixed direction summed across a population of MT neurons that have different PDs evenly spanning $360^{\circ}$. This summed population response is invariant to the direction of the stimulus component, which is suitable for representing signal strength. Equivalently, the summed population neural response in MT can be approximated by summing the responses of each neuron elicited by a stimulus component moving in different directions spanning $360^{\circ}$ and averaged across neurons in our data sample. We calculated $S_{1}$ and $S_{2}$ based on the following equation: $S_{k}=\frac{\sum_{j=1}^{N}\left[\Sigma_{i=1}^{M} R_{k, j}\left(\theta_{i}\right)\right]}{N}$, in which $k$ is the index of the stimulus component. For the low-contrast component, $k=1$; for the high-contrast component, $k=2$; $N$ is the total number of neurons in the population; $R_{k, j}\left(\theta_{i}\right)$ represents the raw firing rate of neuron $j$ to motion direction $\theta_{I}$ of the stimulus component $k ; M$ is the number of the direction samples of a direction tuning curve. Because we spline-fitted the direction tuning curve using a step of $1^{\circ}, M=360$. The values of $S_{1}$ and $S_{2}$ for the two stimulus components are shown in Table 2.

This normalization model (Eq. 5) failed to capture the response tuning to overlapping bidirectional stimuli, accounting for only $32 \%$ of the response variance (32\% for site a, $32 \%$ for site b). The model performed better when stimuli were separated, accounting for $62 \%$ of the variance. We found similar results when using this model to fit the data from our second experiment, in which luminance contrast competed with motion speed. The model accounted for an average of $45 \%$ of the response variance (39\% for site a, $50 \%$ for site b) when stimuli were overlapping, and $76 \%$ of the variance when stimuli were separated (Table 2).

It has been suggested that response normalization can be tuned, such that individual stimulus components contribute differently to normalization (Carandini et al., 1997; Rust et al., 2006; $\mathrm{Ni}$ et al., 2012). Therefore, we fitted our data using a tuned normalization equation:

$R_{\text {pred }}\left(\theta_{1}, \theta_{2}\right)=\frac{S_{1}^{n}}{S_{1}^{n}+\alpha S_{2}^{n}+\sigma} R_{1}\left(\theta_{1}\right)+\frac{S_{2}^{n}}{S_{1}^{n}+\alpha S_{2}^{n}+\sigma} R_{2}\left(\theta_{2}\right)+c$, where $\alpha$ is a positive parameter that scales the contribution of $S_{2}$ with respect to $S_{1}$ to normalization. We found that introducing tuned normalization did not improve the model performance at all when stimuli were overlapping, accounting for an average of $32 \%$ of the response variance ( $32 \%$ for site a, $31 \%$ for site b). When stimuli were separated, the tuned normalization model accounted for $64 \%$ of the variance. We found similar results when fitting the data collected when contrast competed with speed (Table 2).

The poor fit of the responses under the overlapping condition by the standard normalization model (Eq. 5) can be understood because MT neurons showed a very strong bias toward the high-contrast component, whereas $S_{1}$ and $S_{2}$ were similar, with $S_{2}$ (i.e., MT responses elicited by the highcontrast component) being slightly smaller (Table 2). The tuned normalization was not able to improve the fit because, although it changed the relative contributions of the stimulus components to the normalization pool in the denominator, it kept the numerators in Equation 6 unchanged. Hence, the relative weights for the two stimulus components did not change. The failure of the normalization model fit using Equations 5 and 6 under the overlapping condition suggests that the assumption that the response of an MT neuron to multiple overlapping stimuli is governed by the population responses in area MT elicited by the individual stimulus components may be flawed. 
Table 2. Fitting the direction tuning curves using normalization models

\begin{tabular}{|c|c|c|c|c|c|}
\hline Visual stimuli & $S_{1}$ & $\mathrm{~S}_{2}$ & Percentage of & ce accounted for (mean & \\
\hline $\begin{array}{l}\text { Contrast versus coherence } \\
\qquad(N=70)\end{array}$ & Low contrast and high coherence & High contrast and low coherence & $\begin{array}{l}\text { Normalization } \\
\text { (Eq. 5) }\end{array}$ & $\begin{array}{l}\text { Tuned } \\
\text { normalization (Eq. 6) }\end{array}$ & $\begin{array}{l}\text { Normalization with weighted } \\
\text { numerators (Eq. } 7 \text { ) }\end{array}$ \\
\hline Overlapping (site a) & 7650 & 7574 & $32 \pm 18$ & $32 \pm 18$ & $80 \pm 20$ \\
\hline Overlapping (site b) & 7589 & 7505 & $32 \pm 18$ & $31 \pm 19$ & $71 \pm 25$ \\
\hline Spatially separated & 7589 & 7574 & $62 \pm 22$ & $64 \pm 23$ & $76 \pm 21$ \\
\hline $\begin{array}{l}\text { Contrast versus speed } \\
\qquad(N=13)\end{array}$ & Low contrast and faster speed & High contrast and slower speed & & & \\
\hline Overlapping (site a) & 7889 & 6202 & $39 \pm 21$ & $38 \pm 21$ & $86 \pm 15$ \\
\hline Overlapping (site b) & 7174 & 6008 & $50 \pm 20$ & $49 \pm 20$ & $78 \pm 19$ \\
\hline Spatially separated & 7174 & 6202 & $76 \pm 19$ & $77 \pm 20$ & $88 \pm 5$ \\
\hline
\end{tabular}

To capture the strong bias toward the high-contrast component in the overlapping condition, a weighting parameter is needed in the numerator. Accordingly, we fitted our results using the following equation:

$$
R_{\text {pred }}\left(\theta_{1}, \theta_{2}\right)=\frac{S_{1}^{n}}{S_{1}^{n}+\beta S_{2}^{n}+\sigma} R_{1}\left(\theta_{1}\right)+\frac{\beta S_{2}^{n}}{S_{1}^{n}+\beta S_{2}^{n}+\sigma} R_{2}\left(\theta_{2}\right)+c,
$$

where $\beta$ is a positive parameter and appears in both the numerator and the denominator. This parameter allows the relative response weights for the two stimulus components to vary. When $\beta$ is $>1$, the response weight for the high-contrast component $\left(R_{2}\right)$ is greater than that for the low-contrast component $\left(R_{1}\right)$. As expected, this equation fitted the data well, accounting for $>70 \%$ of the response variance for both the overlapping and spatially separated conditions (Table 2). However, the normalization model itself does not provide an explanation for why the response weight is greater for the high-contrast component in the overlapping condition but not in the spatially separated condition. Our interpretation of the success of this model fit is that the term $\beta$ in Equation 7 serves to capture the relative signal strength of the two stimulus components, such that the signal strength of the highcontrast and low-coherence (or low-speed) component is greater than that of the low-contrast and high-coherence (or high-speed) component under the overlapping but not spatially separated condition. Equation 7 is similar to a tuned normalization model used by Ni and colleagues (Ni et al., 2012; Ni and Maunsell, 2017, 2019) to explain the effect of attention on the neuronal response to multiple stimuli within the RF. In Equations $3 \mathrm{~A}$ and $3 \mathrm{~B}$ of $\mathrm{Ni}$ et al. (2012), there is also a term $\beta$ in the numerator, which increases the weight of the attended stimulus component. Although $\beta$ in the numerator can reflect attentional modulation as shown in these previous studies, $\beta$ can also represent biased feedforward drive that favors one stimulus component under the overlapping condition. Our model simulations, presented in the following section, support the latter possibility. We will further consider the potential involvement of attention in the Discussion.

\section{Computer simulations using a V1-MT model}

Our spatially separated visual stimuli fall inside the RFs of single MT neurons, whereas only one of the stimulus components would fall inside the RFs of single V1 neurons. Hence, our spatially separated visual stimuli can interact within the RFs of MT neurons but not V1 neurons. In contrast, the overlapping stimuli can interact within the RFs of both MT and V1 neurons. To explore the neural mechanisms underlying our physiological findings, we conducted computer simulations using a hierarchi- cal feedforward model adapted from Simoncelli and Heeger (1998). This model consists of two processing stages corresponding to areas V1 and MT. Each stage carries out a series of computations, including spatiotemporal filtering, spatial pooling, rectification, and divisive normalization. At the V1 stage, simple cells receive input directly from the visual stimulus, and complex cells pool inputs from rectified and divisively normalized responses of V1 simple cells. At the MT stage, MT neurons pool inputs from V1 complex cells, followed by rectification and divisive normalization (Simoncelli and Heeger, 1998; Rust et al., 2006).

We generated random-dot visual stimuli that are similar to those used in our physiological experiments with the same size and dot density and simulated the neuronal responses in areas MT and V1. The visual stimuli and a simplified architecture of the model are illustrated in Figure 7. The diameter of each randomdot patch was $3^{\circ}$, extending 63 pixels. The dot density of each random-dot patch was 2.7 dots/degree ${ }^{2}$. The RF sizes of model V1 and MT neurons, set by the sizes of the convolution filters, were $1.2^{\circ}$ and $10^{\circ}$ in diameter, respectively (see Materials and Methods). The populations of model neurons in V1 and MT stages approximately tiled a sphere in the spatiotemporal frequency domain, as in the Simoncelli and Heeger (1998) model. The RFs of V1 and MT neuron populations covered a region of the visual field that was $17.3^{\circ} \times 17.3^{\circ}$. In the overlapping condition, the apertures of two random-dot patches overlapped within the RFs (Fig. 7A). In the spatially separated condition, the two random-dot patches were placed side by side, separated by a blank gap that was $1.2^{\circ}$ wide, within the RFs of single MT neurons (Fig. $7 B$ ). In the overlapping condition, the $\mathrm{V} 1$ neurons whose RFs covered site a were activated by both stimulus components (Fig. 7A). In the spatially separated condition, V1 neurons were activated by only one stimulus component, either at site a or site b (Fig. 7B).

We tuned the model parameters (see Materials and Methods) to match the experimentally measured contrast response functions of V1 and MT neurons (Sclar et al., 1990) and the coherence response function of MT neurons (Britten and Newsome, 1998). The simulated contrast response functions of V1 and MT neurons fitted the experimental data almost perfectly, and the simulated coherence response function of MT neurons matched the data reasonably well (Fig. $8 A-C$ ). As far as we know, an experimentally measured coherence response function of $\mathrm{V} 1$ neurons has not been described previously. Our simulations show that V1 responses increased with the coherence level of moving randomdot stimuli (Fig. 8D). The model V1 neurons had slightly higher firing rates in response to low-coherence stimuli and, as expected, 
A

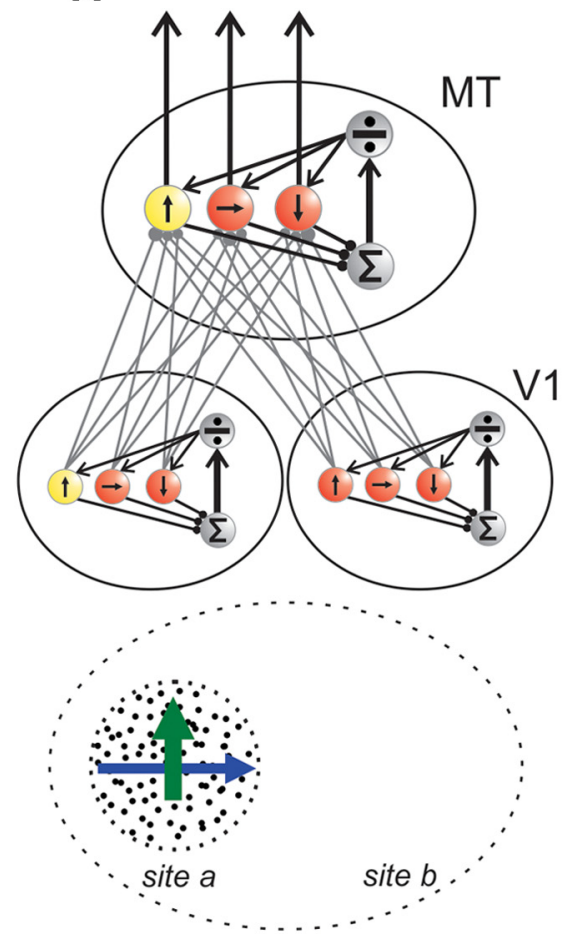

B

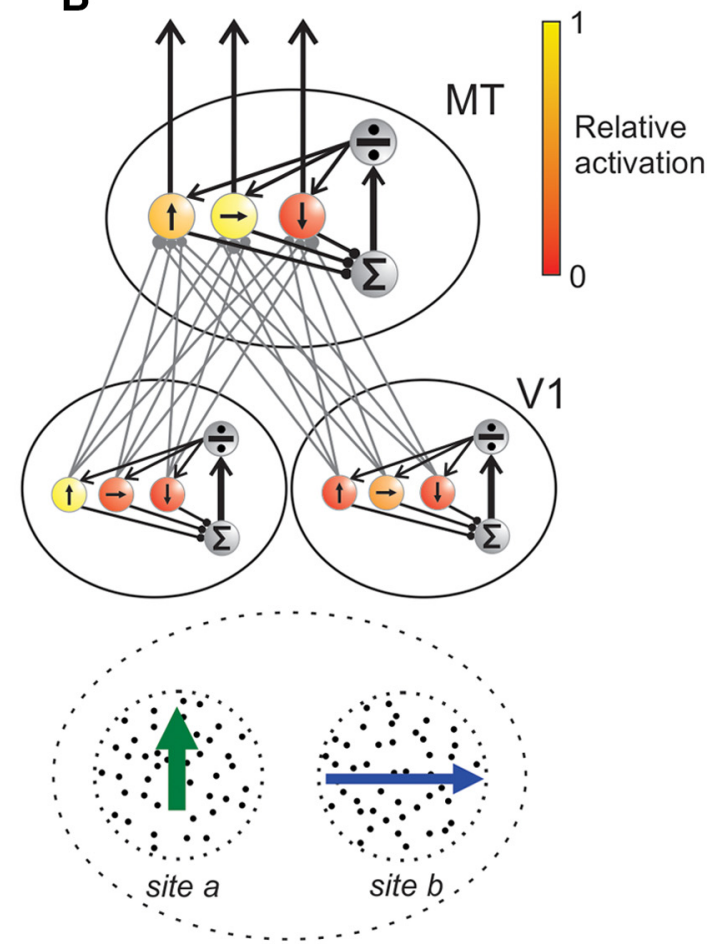

Figure 7. Illustration of a simplified architecture for the V1-MT model. Each MT neuron receives feedforward inputs from multiple neurons at the V1 stage. Responses are divisively normalized by the sum of local population activity at both V1 and MT stages. Each small circle represents a neuron, and the black arrow inside the circle indicates the PD. The color of each circle indicates the response magnitude of the neuron. Yellow means maximum response and red means minimum response. Visual stimuli are illustrated below the neural circuit as the input to the V1 stage. The green and blue arrows represent the high-contrast and low-coherence component and the low-contrast and high-coherence component, respectively. Two pools of neurons at the V1 stage that respond only to site a or site b, respectively, are illustrated. The RFs of the MT neurons are illustrated by the dotted ellipses and cover both site a and site b. $\boldsymbol{A}, 0$ verlapping condition. $\boldsymbol{B}$, Spatially separated condition.

more trial-to-trial variability in comparison with the model MT neurons due to small V1 RFs (Fig. 8C,D).

The MT responses elicited by our visual stimuli that competed between luminance contrast and motion coherence were well captured by the model. Consistent with our experimental data (Fig. 2), the tuning curve of model MT neurons to the lowcontrast and high-coherence component had a greater peak response than that of the high-contrast and low-coherence component (Fig. 9A,B). In the overlapping condition, the simulated MT response elicited by the bidirectional stimuli was nearly completely biased toward the weaker high-contrast and lowcoherence component (Fig. 9A), as found in the neural data. The model also captured the change of MT response tuning when visual stimuli were rearranged spatially. In the spatially separated condition, the tuning curve of model MT neurons elicited by the bidirectional stimuli was no longer dominated by the highcontrast and low-coherence component (Fig. 9B).

At the V1 stage of the model, the tuning curves of V1 complex cells showed a slightly greater mean peak response to the highcontrast and low-coherence component than to the low-contrast and high-coherence component (Fig. 9C). In the overlapping condition, the simulated $\mathrm{V} 1$ response elicited by the bidirectional stimuli was strongly biased toward the high-contrast and lowcoherence component (Fig. 9C), to an extent similar to that found in model MT neurons (Fig. 9A), as measured by the weights for the component responses using the SNL model fits. The bias index (Eq. 4) for the V1 model neurons was 0.77, and that for the MT model neurons was 0.84 . These simulation results suggest that the strong bias toward the high-contrast and low- coherence component found in MT neurons is inherited from V1.

In the spatially separated condition, the V1 response elicited by the bidirectional stimuli was the same as that elicited by the single stimulus component placed within the RFs of V1 neurons (Fig. 9D,E). Although the V1 peak response elicited by the highcontrast and low-coherence component at site a was slightly stronger than that elicited by the low-contrast and highcoherence component at site b, the MT response elicited by the bidirectional stimuli was skewed toward the low-contrast and high-coherence component, consistent with the average of the component responses (Fig. 9B). These simulation results suggest that MT response elicited by the bidirectional stimuli in the spatially separated condition (Fig. 9B) may be due to feature competition within MT.

The dot density of overlapping stimuli was twice the density of a single stimulus in the spatially separated condition. To understand whether the change of stimulus competition with the spatial arrangement of visual stimuli was confounded by the dot density, we conducted the same model simulations using a dot density that was either half of (1.35 dots/degree $\left.{ }^{2}\right)$ or twice (5.4

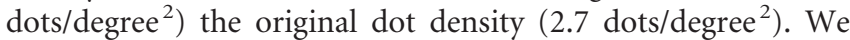
found essentially the same results using these three different dot densities (results not shown), suggesting that our findings cannot be explained by the difference of dot density under the overlapping and spatially separated conditions.

The response tuning curves of single MT neurons measured by varying the VA direction of the bidirectional stimuli can be mapped to the responses of a population of MT neurons that 

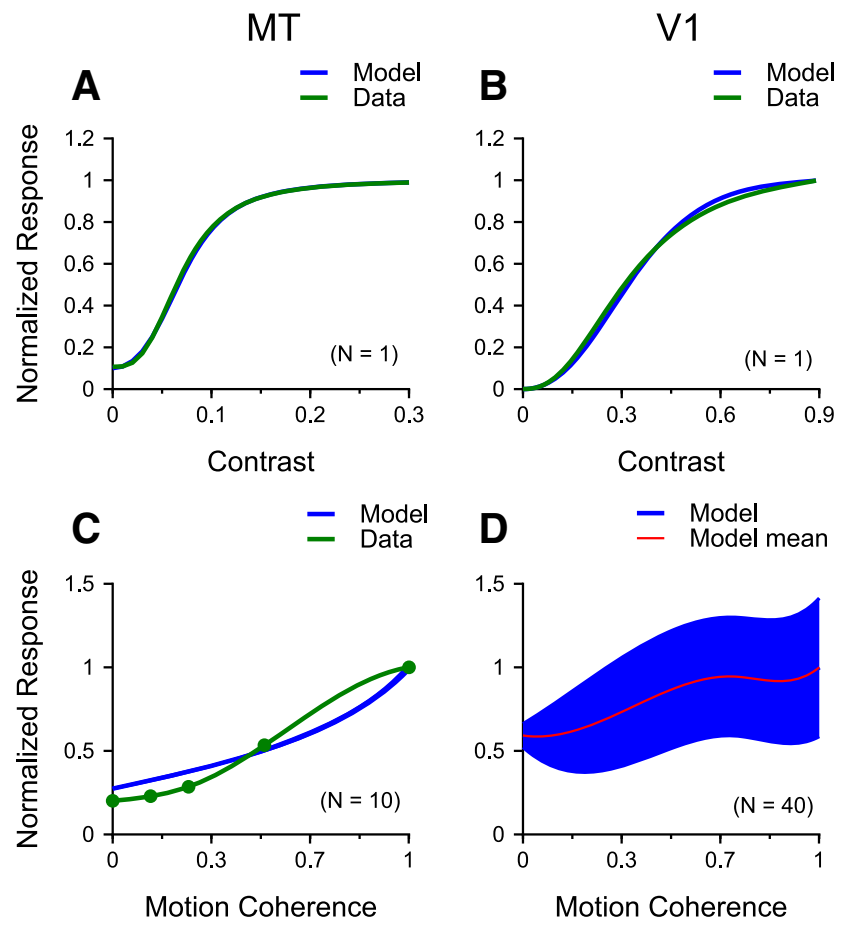

Figure 8. $\boldsymbol{A}-\boldsymbol{D}$, Contrast and coherence response functions of model $\mathrm{V} 1(\boldsymbol{B}, \boldsymbol{D})$ and $\mathrm{MT}(\boldsymbol{A}, \boldsymbol{C})$ neurons. $\boldsymbol{A}, \boldsymbol{B}$, Contrast response functions fitted to sinusoidal gratings for model neurons. Green curves are experimental data replotted from Sclar et al. (1990). C, Coherence response function fitted to high-contrast random dots for model MT neurons. Green dots are experimental data replotted from Britten and Newsome (1998). The green curve is the spline fit of the experimental data points. $\boldsymbol{D}$, Coherence response to high-contrast random dots for model V1 complex cells. $\boldsymbol{C}, \boldsymbol{D}$, The widths of the blue curves represent the SD. $N$ indicates the number of repeats for simulations. The stimulus dots were regenerated randomly for each simulation in $C$ and $\boldsymbol{D}$.

have different PDs, elicited by the bidirectional stimuli moving in a given VA direction. Figure 7 summarizes the changes of the response distributions across neuron populations at V1 and MT stages under the overlapping and spatially separated conditions.

To determine whether our findings can be generalized to visual stimuli other than random dots, we conducted model simulations using sinusoidal gratings that had different luminance contrasts and spatial frequencies (SFs). Because V1 and MT neurons have heterogeneous SF selectivity, we set SF of the visual stimuli in relation to the preferred SF of the model MT neuron. We chose the stimulus parameters such that one grating component had a "high-contrast and less-preferred SF," whereas the other grating component had a "low-contrast and preferred SF." The two grating components drifted at the same temporal frequency in two directions separated by $90^{\circ}$ and were either overlapping or spatially separated within the RF of an MT neuron. Except for visual stimuli, all other model parameters were identical to those in our model simulations using random-dot stimuli.

We found the same results using drifting gratings as those obtained using random-dot stimuli (Fig. 10). The model MT neuron showed a strong response bias toward the high-contrast and less-preferred SF component when two grating components were overlapping (Fig. 10A), even though the MT response elicited by the high-contrast and less-preferred SF component presented alone (Fig. 10A, green curve) was significantly weaker than that elicited by the low-contrast and preferred SF component (Fig. 10A, blue curve). The strong bias toward the high-contrast and less-preferred SF grating was abolished when the two gratings were spatially separated (Fig. 10B).

In the model, a given MT neuron pools the inputs from a group of V1 complex cells. The model V1 neuron shown in Figure 10 had the same preferred SF as the model MT neuron. The direction tuning curves of this V1 neuron showed greater response to the high-contrast and less-preferred SF component than to the low-contrast and preferred SF component (Fig. 10C). In the overlapping condition, the simulated V1 response elicited by the bidirectional stimuli was strongly biased toward the highcontrast and less-preferred SF grating, completely ignoring the low-contrast and preferred SF grating (Fig. 10C). When gratings were spatially separated, the V1 neuron only responded to one grating component (Fig. 10D,E). These simulation results further support the idea that the strong bias toward the highcontrast component found in MT under the overlapping condition is inherited from V1. Our model simulations predict that MT neurons may inherit stimulus preferences of V1 neurons across a wide range of feature domains when multiple stimuli compete within the RFs of V1 neurons. Together, our results reveal the importance of neural processing at different stages of the visual hierarchy in determining how multiple visual stimuli compete within RFs of neurons in a given brain area.

\section{Discussion}

We have shown that the way MT neurons represent multiple stimuli competing in more than one feature domain depends on the spatial arrangement of the visual stimuli. When two stimuli are overlapping, MT responses are dominated by the stimulus component that has high contrast. When two stimuli are spatially separated, the contrast dominance is abolished. Our neural data and model simulations suggest that the contrast dominance found with overlapping stimuli is due to normalization occurring at an input stage fed to MT, and MT neurons cannot overturn this contrast dominance based on their own feature selectivity. The interaction between spatially separated stimuli can largely be explained by normalization within area MT. By using multiple visual stimuli competing in more than one feature domain, our study revealed how neural processing along the hierarchical visual pathway shapes neural representation of multiple visual stimuli in extrastriate cortex.

\section{Consideration of the effect of attention}

Attention can bias neuronal responses elicited by multiple stimuli in the RF in favor of the attended stimulus (Treue and Maunsell, 1996; Ferrera and Lisberger, 1997; Reynolds et al., 1999; Treue and Martínez Trujillo, 1999; Recanzone and Wurtz, 2000; Li and Basso, 2005; Lee and Maunsell, 2010). Although the animals in this study performed a fixation task without the need to engage goal-directed attention, could the high-contrast component capture stimulus-driven attention (Corbetta and Shulman, 2002) and bias the neuronal response elicited by the overlapping stimuli? Several considerations argue against this possibility. $\mathrm{Al}$ though an abrupt stimulus onset captures attention (Yantis and Jonides, 1984), a visual stimulus that is brighter than other distractors does not automatically capture attention (Jonides and Yantis, 1988). The two stimulus components of our overlapping stimuli were turned on and started to move at the same time. The stimulus onset may automatically draw attention toward the spatial location of the overlapping stimuli, but it is unlikely to draw attention toward only the high-contrast component. Furthermore, stimulus-driven attention occurs with a time delay (Nakayama and Mackeben, 1989) and its effect on neuronal re- 


\section{Overlapping}

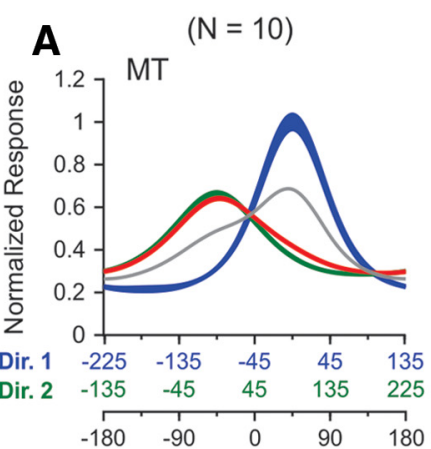

C

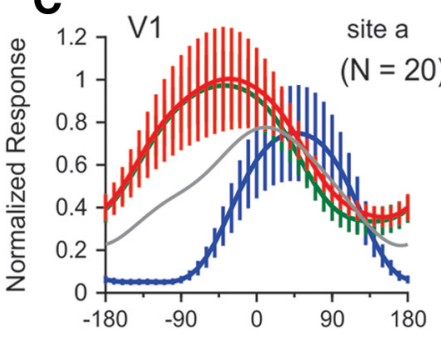

Vector Average Direction (deg)

\section{Spatially Separated}

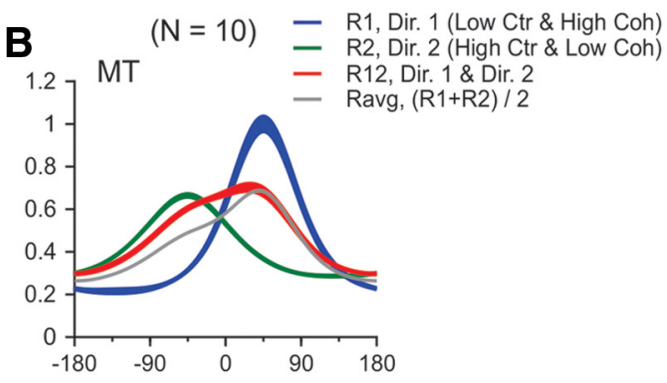

Vector Average Direction (deg)

D

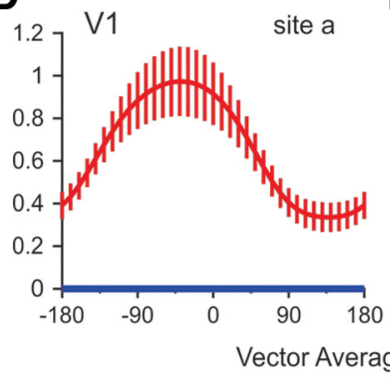

E

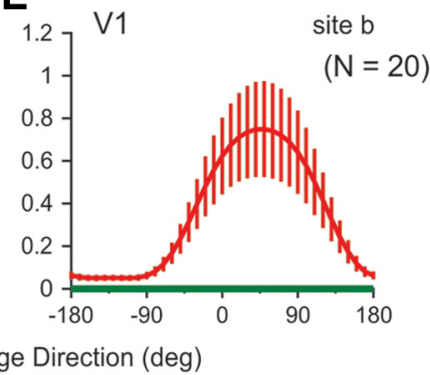

Figure 9. Computer simulations of direction tuning curves of MT and V1 neurons to the bidirectional stimuli used in the main physiological experiment. $\boldsymbol{A}-\boldsymbol{E}$, The visual stimuli are either overlapping $(\boldsymbol{A}, \boldsymbol{C})$ or spatially separated $(\boldsymbol{B}, \boldsymbol{D}, \boldsymbol{E})$ within the RFs of model MT neurons. The two stimulus components compete in luminance contrast and motion coherence. The simulated responses to the low-contrast and high-coherence component and the high-contrast and low-coherence component are shown in blue and green, respectively. The responses to the bidirectional stimuli are shown in red. $\boldsymbol{A}$, The vector average direction and the directions of individual stimulus components are labeled in the corresponding $x$-axes, following the same convention as in Figure 2 . $A, B$, Simulated responses of model MT neurons. $\boldsymbol{C}-\boldsymbol{E}$, Simulated responses of model V1 complex cells. $\boldsymbol{A}-\boldsymbol{E}$, Widths of the tuning curves $(\boldsymbol{A}$ and $\boldsymbol{B})$ and the error bars $(\boldsymbol{C}-\boldsymbol{E})$ represent SDs.

\section{Overlapping}

\section{A}

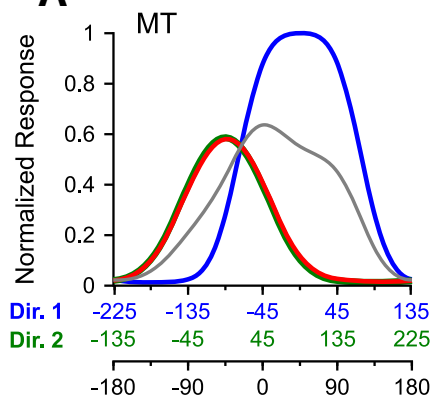

C

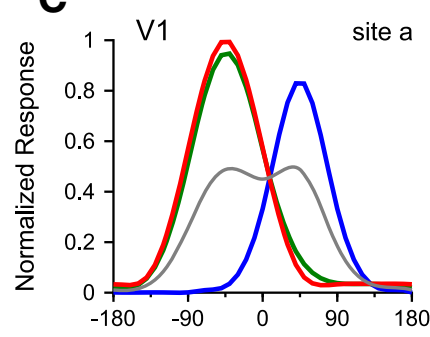

site a

Vector Average Direction (deg)

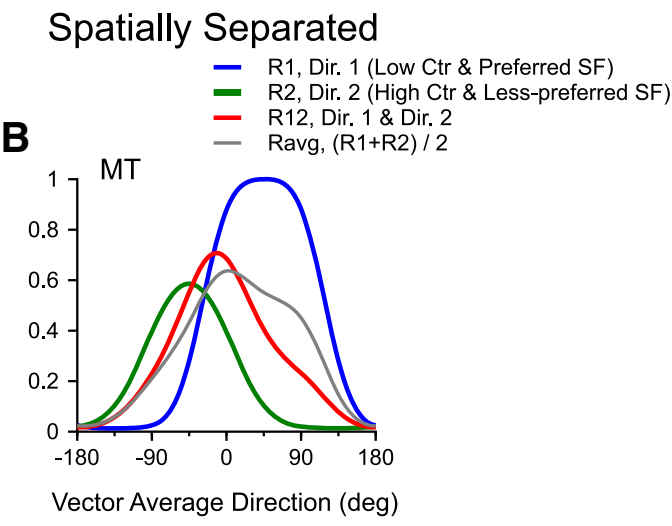

D

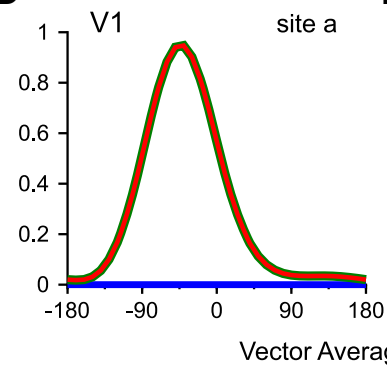

$\mathbf{E}$

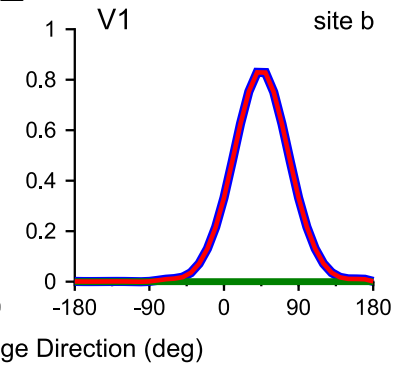

Figure 10. Computer simulations of direction tuning curves of MT and V1 neurons to drifting sinusoidal gratings that competed in luminance contrast and SF. $\boldsymbol{A}-\boldsymbol{E}$, The two gratings were either overlapping $(\boldsymbol{A}, \boldsymbol{C})$ or spatially separated $(\boldsymbol{B}, \boldsymbol{D}, \boldsymbol{E})$ within the RF of the MT neuron. The preferred SF of the model MT and V1 neurons was 2 cycles/deg. The high-contrast less-preferred SF component had a contrast of 0.77 and an SF of 1 cycle/deg. The low-contrast and preferred SF component had a contrast of 0.36 and an SF of 2 cycle/deg. The grating components drifted at the same temporal frequency of 9.2 cycles/s in two directions separated by $90^{\circ}$. The simulated responses to the low-contrast and preferred SF component and the high-contrast and less-preferred SF component are shown in blue and green, respectively. The responses to the bidirectional stimuli are shown in red. $A$, The vector average direction and the directions of individual grating components are labeled in the corresponding $x$-axes, following the same convention as in Figure 9. $A, B$, Simulated responses of a model MT neuron. $C-E$, Simulated responses of a model V1 complex cell. 
sponses in MT is transient, lasting for $\sim 70 \mathrm{~ms}$ (Busse et al., 2008). In contrast, we found that the response bias toward the highcontrast component is present at the very beginning of the neuronal responses following the stimulus onset, and the bias is very stable and persistent throughout the motion period (Fig. 5). In addition, Wannig et al. (2007) have shown that attention directed to one of two overlapping surfaces can alter the responses of MT neurons. However, the effect of the surface-based attention was also delayed, and attention led to a response magnitude modulation of $\sim 20 \%$ in MT between conditions when attention was directed to two different surfaces (Wannig et al., 2007). Even in the unlikely case that the animals were consistently attending to the high-contrast component throughout the stimulus presentation period in our study, the effect of attention would be insufficient to account for the instant and nearly complete dominance by the high-contrast component.

\section{Mechanisms underlying stimulus interactions}

The primate visual system is hierarchically organized (Maunsell and van Essen, 1983; Felleman and Van Essen, 1991). The response properties of neurons in a visual area are shaped by feedforward input, as well as intra-areal and feedback processes. To understand the mechanisms underlying neural encoding of multiple stimuli, it is important to determine how these processes contribute to the RF properties in a given visual area. However, it is often difficult to disentangle the contribution of feedforward input from other neural processes. We have previously found that, in response to overlapping stimuli, MT neurons show a bias toward the stimulus component that has a higher signal strength, defined by either luminance contrast or motion coherence (Xiao et al., 2014). The response bias can be described by a model of divisive normalization. Because neurons in $\mathrm{V} 1$ also show a bias toward the stimulus component that has a higher contrast (Busse et al., 2009; MacEvoy et al., 2009) and divisive normalization may occur in both V1 and MT (Simoncelli and Heeger, 1998; Heuer and Britten, 2002), it was unclear how the feedforward input from V1 contributed to the response bias found in MT.

In this study, we were able to differentiate the impact of feedforward input from other neural processes on the response properties of MT neurons. Our results suggest that neurons in V1 may respond more strongly to the high-contrast and low-coherence component than to the low-contrast and high-coherence component used in our experiment due to the sensitivity of V1 neurons to contrast and coherence. When two stimuli overlap, the responses of V1 neurons elicited by both stimulus components may already show a strong bias toward the high-contrast and low-coherence component due to divisive normalization in V1 (Fig. 9C). MT neurons are no longer able to remix the stimulus components according to their own sensitivities to contrast and coherence. In other words, MT neurons inherit the response bias toward the high-contrast component from their input. When two visual stimuli are spatially separated, MT neurons receive inputs from two different pools of V1 neurons, and each neuron pool responds to only one stimulus component (Fig. $7 B$ ). The neuronal responses elicited by the two stimulus components remain separated in V1. MT neurons can mix the responses elicited by the two stimulus components via spatial and directional pooling and divisive normalization within MT. As a result, the mixing in the MT may well reflect the sensitivities of MT neurons to different stimulus features. Our model simulations make predictions regarding how V1 and MT neurons respond to multiple competing stimuli (e.g., as shown in Figs. 9C, 10), which can be tested in a future physiological study.

\section{Implications on normalization and encoding of multiple visual stimuli}

Our finding that the response weighting for competing stimuli depends on the spatial arrangement provides a new perspective on the well established normalization model (Carandini and Heeger, 2011). The basic form of normalization equations (Eqs. 5,6 ) predicts that the response weight for a stimulus component increases with its signal strength, but does not consider the spatial arrangement of the visual stimuli. We made a surprising finding that MT response to overlapping stimuli cannot be predicted by the population neural responses in MT elicited by the individual stimulus components. One must consider the neural computations occurring along the hierarchical visual pathway. Majaj et al. (2007) showed that pattern direction-selective neurons in MT characterized by overlapping drifting gratings (i.e., plaid) did not integrate the directions of the component gratings when they were spatially separated within the RF, suggesting that the computation underlying pattern direction selectivity in MT is local. Different from the plaid, the overlapping random-dot stimuli used in our study elicit the percept of motion transparency. We showed that changing the spatial arrangement of visual stimuli can have a substantial impact not only on motion integration but also on the competition between multiple stimuli. Our results revealed that contrast has a dominant effect in determining stimulus competition within a local spatial region when multiple stimuli differ in more than one feature domain. When visual stimuli are spatially separated, the effect of contrast is substantially reduced.

A seminal model involving MT neurons pooling inputs from V1 and divisive normalization in both V1 and MT has been successful in explaining a range of experimental results of MT responses (Simoncelli and Heeger, 1998; Rust et al., 2006). However, the model in its original form does not specify how features are spatially integrated, and it does not differentiate overlapping and spatially separated stimuli (Majaj et al., 2007). In our study, we adapted this model to simulate both overlapping and spatially separated conditions and showed that the framework can explain our main physiological findings. Also using this model, Busse et al. (2009) previously demonstrated the impact of response normalization in V1 on neural response in MT. They showed that, by making the contrasts of two drifting gratings of a plaid to be unequal, the response of a model MT neuron changed from representing the pattern motion of the plaid to mostly representing the higher-contrast grating component, likely due to contrast normalization in V1 (Busse et al., 2009). However, the MT response elicited by the higher-contrast grating alone could also be greater than that elicited by the lower-contrast grating. Response normalization within MT may also contribute to the modelpredicted response bias toward the higher-contrast component in MT, akin to our experimental result obtained using randomdot stimuli with unequal contrasts (Xiao et al., 2014). In comparison, our current study provides unequivocal new evidence on how responses in MT are shaped by the hierarchical network. By using two stimuli competing in more than one feature domain, we demonstrated neurophysiologically and computationally the substantial impact of stimulus competition in the input stage on the neuronal responses in MT and how that impact changes with the spatial arrangement of visual stimuli. Our findings may also apply to other visual areas in the hierarchical network, including 
those in the ventral visual stream where response normalization has been well documented.

\section{References}

Albright TD, Desimone R (1987) Local precision of visuotopic organization in the middle temporal area (MT) of the macaque. Exp Brain Res 65: 582-592.

Bao P, Tsao DY (2018) Representation of multiple objects in macaque category-selective areas. Nat Commun 9:1774.

Born RT, Bradley DC (2005) Structure and function of visual area MT. Annu Rev Neurosci 28:157-189.

Braddick O (1993) Segmentation versus integration in visual motion processing. Trends Neurosci 16:263-268.

Britten KH (2003) The middle temporal area: motion processing and the link to perception. In: The visual neurosciences (Chalupa LM, Werner JS, eds), pp 1203-1216. London: MIT.

Britten KH, Heuer HW (1999) Spatial summation in the receptive fields of MT neurons. J Neurosci 19:5074-5084.

Britten KH, Newsome WT (1998) Tuning bandwidths for near-threshold stimuli in area MT. J Neurophysiol 80:762-770.

Britten KH, Shadlen MN, Newsome WT, Movshon JA (1992) The analysis of visual motion: a comparison of neuronal and psychophysical performance. J Neurosci 12:4745-4765.

Britten KH, Shadlen MN, Newsome WT, Movshon JA (1993) Responses of neurons in macaque MT to stochastic motion signals. Vis Neurosci 10:1157-1169.

Busse L, Katzner S, Treue S (2008) Temporal dynamics of neuronal modulation during exogenous and endogenous shifts of visual attention in macaque area MT. Proc Natl Acad Sci U S A 105:16380-16385.

Busse L, Wade AR, Carandini M (2009) Representation of concurrent stimuli by population activity in visual cortex. Neuron 64:931-942.

Carandini M, Heeger DJ (2011) Normalization as a canonical neural computation. Nat Rev Neurosci 13:51-62.

Carandini M, Heeger DJ, Movshon JA (1997) Linearity and normalization in simple cells of the macaque primary visual cortex. J Neurosci 17 : 8621-8644.

Corbetta M, Shulman GL (2002) Control of goal-directed and stimulusdriven attention in the brain. Nat Rev Neurosci 3:201-215.

Felleman DJ, Van Essen DC (1991) Distributed hierarchical processing in the primate cerebral cortex. Cereb Cortex 1(1):1-47.

Ferrera VP, Lisberger SG (1997) Neuronal responses in visual areas MT and MST during smooth pursuit target selection. J Neurophysiol 78:14331446.

Gattass R, Gross CG (1981) Visual topography of striate projection zone (MT) in posterior superior temporal sulcus of the macaque. J Neurophysiol 46:621-638.

Heuer HW, Britten KH (2002) Contrast dependence of response normalization in area MT of the rhesus macaque. J Neurophysiol 88:3398-3408.

Jonides J, Yantis S (1988) Uniqueness of abrupt visual onset in capturing attention. Percept Psychophys 43:346-354.

Lee J, Maunsell JH (2010) Attentional modulation of MT neurons with single or multiple stimuli in their receptive fields. J Neurosci 30:3058-3066.

Li X, Basso MA (2005) Competitive stimulus interactions within single response fields of superior colliculus neurons. J Neurosci 25:11357-11373.

MacEvoy SP, Tucker TR, Fitzpatrick D (2009) A precise form of divisive suppression supports population coding in the primary visual cortex. Nat Neurosci 12:637-645.

Majaj NJ, Carandini M, Movshon JA (2007) Motion integration by neurons in macaque MT is local, not global. J Neurosci 27:366-370.
Maunsell JH, van Essen DC (1983) The connections of the middle temporal visual area (MT) and their relationship to a cortical hierarchy in the macaque monkey. J Neurosci 3:2563-2586.

McDonald JS, Clifford CW, Solomon SS, Chen SC, Solomon SG (2014) Integration and segregation of multiple motion signals by neurons in area MT of primate. J Neurophysiol 111:369-378.

Morgan ML, Deangelis GC, Angelaki DE (2008) Multisensory integration in macaque visual cortex depends on cue reliability. Neuron 59:662-673.

Movshon JA, Newsome WT (1996) Visual response properties of striate cortical neurons projecting to area MT in macaque monkeys. J Neurosci 16:7733-7741.

Nakayama K, Mackeben M (1989) Sustained and transient components of focal visual attention. Vision Res 29:1631-1647.

Newsome WT, Paré EB (1988) A selective impairment of motion perception following lesions of the middle temporal visual area (MT). J Neurosci 8:2201-2211.

Ni AM, Maunsell JHR (2017) Spatially tuned normalization explains attention modulation variance within neurons. J Neurophysiol 118:19031913.

Ni AM, Maunsell JHR (2019) Neuronal effects of spatial and feature attention differ due to normalization. J Neurosci 39:5493-5505.

Ni AM, Ray S, Maunsell JH (2012) Tuned normalization explains the size of attention modulations. Neuron 73:803-813.

Park WJ, Tadin D (2019) Motion perception. In: Stevens' handbook of experimental psychology and cognitive neuroscience. (Wixted JT, ed). New York: Wiley.

Qian N, Andersen RA (1994) Transparent motion perception as detection of unbalanced motion signals. II. Physiology. J Neurosci 14:7367-7380.

Recanzone GH, Wurtz RH (2000) Effects of attention on MT and MST neuronal activity during pursuit initiation. J Neurophysiol 83:777-790.

Recanzone GH, Wurtz RH, Schwarz U (1997) Responses of MT and MST neurons to one and two moving objects in the receptive field. J Neurophysiol 78:2904-2915.

Reynolds JH, Chelazzi L, Desimone R (1999) Competitive mechanisms subserve attention in macaque areas V2 and V4. J Neurosci 19:1736-1753.

Rust NC, Mante V, Simoncelli EP, Movshon JA (2006) How MT cells analyze the motion of visual patterns. Nat Neurosci 9:1421-1431.

Sclar G, Maunsell JH, Lennie P (1990) Coding of image contrast in central visual pathways of the macaque monkey. Vision Res 30:1-10.

Simoncelli EP, Heeger DJ (1998) A model of neuronal responses in visual area MT. Vision Res 38:743-761.

Snowden RJ, Treue S, Erickson RG, Andersen RA (1991) The response of area MT and V1 neurons to transparent motion. J Neurosci 11:27682785.

Treue S, Martínez Trujillo JC (1999) Feature-based attention influences motion processing gain in macaque visual cortex. Nature 399:575-579.

Treue S, Maunsell JH (1996) Attentional modulation of visual motion processing in cortical areas MT and MST. Nature 382:539-541.

Wannig A, Rodríguez V, Freiwald WA (2007) Attention to surfaces modulates motion processing in extrastriate area MT. Neuron 54:639-651.

Xiao J, Huang X (2015) Distributed and dynamic neural encoding of multiple motion directions of transparently moving stimuli in cortical area MT. J Neurosci 35:16180-16198.

Xiao J, Niu YQ, Wiesner S, Huang X (2014) Normalization of neuronal responses in cortical area MT across signal strengths and motion directions. J Neurophysiol 112:1291-1306.

Yantis S, Jonides J (1984) Abrupt visual onsets and selective attention: evidence from visual search. J Exp Psychol Hum Percept Perform 10:601621 . 